INL/EXT-19-55550

\title{
Architecture Improvements and Retesting of the Microscopic Depletion Implementation in the MAMMOTH Reactor Physics Application
}

Olin Calvin ${ }^{1}$, Javier Ortensi ${ }^{2}$, Yaqi Wang ${ }^{3}$, Sebastian Schunert ${ }^{3}$, Sedat Goluoglu ${ }^{1}$ and Mark DeHart ${ }^{2}$

${ }^{1}$ Nuclear Engineering Program University of Florida 1929 Stadium Road, Room 317 Gainesville, Florida 32611-1934

${ }^{2}$ Reactor Physics Design and Analysis Department Idaho National Laboratory

P.O. Box 1625

Idaho Falls, ID 83415-3840

${ }^{3}$ Nuclear Engineering Methods and Development Department Idaho National Laboratory P.O. Box 1625

Idaho Falls, ID 83415-3840

September 2019

INL is a U.S. Department of Energy National Laboratory operated by Battelle Energy Alliance 


\section{DISCLAIMER}

This information was prepared as an account of work sponsored by an agency of the U.S. Government. Neither the U.S. Government nor any agency thereof, nor any of their employees, makes any warranty, expressed or implied, or assumes any legal liability or responsibility for the accuracy, completeness, or usefulness, of any information, apparatus, product, or process disclosed, or represents that its use would not infringe privately owned rights. References herein to any specific commercial product, process, or service by trade name, trade mark, manufacturer, or otherwise, does not necessarily constitute or imply its endorsement, recommendation, or favoring by the U.S. Government or any agency thereof. The views and opinions of authors expressed herein do not necessarily state or reflect those of the U.S. Government or any agency thereof. 


\title{
Architecture Improvements and Retesting of the Microscopic Depletion Implementation in the MAMMOTH Reactor Physics Application
}

\author{
Olin Calvin, Javier Ortensi, Yaqi Wang, \\ Sebastian Schunert, Sedat Goluoglu and Mark DeHart
}

September 2019

Idaho National Laboratory

Nuclear Systems Design and Analysis

Idaho Falls, Idaho 83415

http://www.inl.gov

Prepared for the

U.S. Department of Energy

Office of Nuclear Energy

Under DOE Idaho Operations Office

Contract DE-AC07-05ID14517 


\begin{abstract}
Nuclear fuel experiments conducted at the Transient Reactor Test Facility (TREAT) will be analyzed with the MAMMOTH reactor physics application, currently under development at Idaho National Laboratory. MAMMOTH natively couples with the BISON, RELAP-7, and Rattlesnake applications within the MOOSE framework. The implemented methods allow for simulating the irradiation of fuel in a nuclear reactor from the beginning of life in a nuclear reactor until it is placed in TREAT for fuel testing using the same finite element mesh throughout the analysis retaining a very high level of resolution and fidelity. The calculation of the isotopic distribution in fuel requires the solution to the decay and transmutation equations coupled to the neutron transport equation. This report focuses on three significant improvements to the MAMMOTH micro-depletion capability. First, the depletion architecture within MAMMOTH is modified to improve the flexibility and speed of burnup calculations. The results of various benchmark tests demonstrate that the refactoring of the previously implemented capability does not introduce any regression. The second improvement involves the expansion of the data functionality. MAMMOTH can now read the decay data files used by the Oak Ridge Isotope Generation (ORIGEN) code, thus enabling access to data of more than 1,600 isotopes. The third improvement is the capability to perform depletion at a constant power level which was verified via a comparison to ORIGEN.
\end{abstract}




\section{Contents}

$\begin{array}{lll}1 & \text { Introduction } & 1\end{array}$

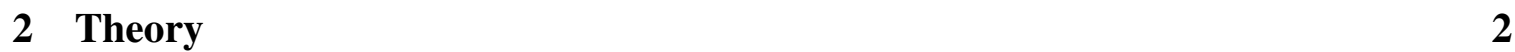

3 The ISOXML Format 4

3.1 Terminology . . . . . . . . . . . . . . . . . . . . 4

3.2 Format . . . . . . . . . . . . . . . . . . . . . 6

3.3 A Sample Valid Library . . . . . . . . . . . . . . . . . . . . . . . . 12

$\begin{array}{lll}4 \text { Data Loader } & 13\end{array}$

$\begin{array}{lll}5 & \text { Architecture } & 18\end{array}$

$6 \quad$ Test Problems $\quad 22$

6.1 Thorium Series . . . . . . . . . . . . . . . . . 22

6.1 .1 MAMMOTH-to-ORIGEN Thorium Series Comparison . . . . . . . 25

6.2 Constant Flux in a Homogeneous System . . . . . . . . . . . . . 26

6.3 MAMMOTH-to-DRAGON5 . . . . . . . . . . . . . . . 27

6.4 MAMMOTH-to-ORIGEN Fuel Pin Comparison . . . . . . . . . . . . . 27

$\begin{array}{llr}7 & \text { Results } & 29\end{array}$

7.1 Thorium Series . . . . . . . . . . . . . . . . . . . . 29

7.1.1 MAMMOTH-to-ORIGEN Thorium Series Comparison . . . . . . . 32

7.2 Constant Flux in a Homogeneous System . . . . . . . . . . . . . . 33

7.3 MAMMOTH-to-DRAGON5 Fuel Pin Comparison . . . . . . . . . . . . . 35

7.4 MAMMOTH-to-ORIGEN Fuel Pin Comparison . . . . . . . . . . . . . . . 42

8 Conclusions 446

\begin{tabular}{ll}
\hline References & 48
\end{tabular} 


\section{Introduction}

The Transient Reactor Test Facility (TREAT) provides the capability of testing traditional and new nuclear fuels concepts under postulated accident conditions. TREAT experiments will encompass both fresh and irradiated fuel. Accurate prediction of the behavior of irradiated fuel in a TREAT experiment requires knowledge of its isotopic composition which in turn requires detailed information of irradiation history, namely local power densities and flux levels.

Idaho National Laboratory uses the MAMMOTH reactor physics code [1] for the analysis of TREAT experiments, because it provides high fidelity multiphysics capabilities for the modeling of nuclear fuel. MAMMOTH is the MOOSE-based [2, 3] reactor physics application that natively couples with the fuel performance code BISON [4], the thermal fluids code RELAP-7 [5] and the neutron transport code Rattlesnake [6, 7]. The multiphysics capabilities of MAMMOTH have been demonstrated for a full size LWR pin that was depleted over several months and then subjected to a Station Black Out (SBO) accident condition [8]. MAMMOTH will allow tracking the isotopic composition of fuel from beginning of life to placing it in TREAT for fuel testing. The great advantage of MAMMOTH over traditional analysis methods is the high fidelity approach of using the same finite element mesh throughout the analysis of irradiation and TREAT testing with the ability to track thousands of isotopes.

The initial implementation and testing of the micro-depletion solver in MAMMOTH is described in a previous report [9]. This work documents the development of the ISOXML format for storing radioactive decay and fission product yield data to be used by MAMMOTH as well as the ability to read ORIGEN ENDF decay data files including the necessary data loader to add this functionality to MAMMOTH. This allows MAMMOTH to execute nuclear transmutation problems with the same data set as ORIGEN (in excess of 1,600 isotopes). Because of changes to the architecture of depletion functionality in MAMMOTH, previous tests used to determine the accuracy of MAMMOTH were executed again to ensure no regression was introduced. In addition, the ability for MAMMOTH to perform depletion at constant power levels was also implemented and a test performed to verify this implementation. 


\section{Theory}

The equation that describes the time rate of change of the $i^{\text {th }}$ nuclide in a material due to nuclear decay is know as the Bateman Equation [10] and is formulated as:

$$
\frac{d N_{i}}{d t}=\sum_{j=1}^{J} \lambda_{j} N_{j}(t) \beta_{j \rightarrow i}-\lambda_{i} N_{i}(t)
$$

where

$J$ is the number of parent nuclides that produce the $i^{\text {th }}$ nuclide

$N_{i}$ is the concentration of the $i^{t h}$ nuclide

$\lambda_{j}$ is the decay constant of the $j^{t h}$ parent nuclide

$\lambda_{i}$ is the decay constant of the $i^{\text {th }}$ nuclide

$\beta_{j \rightarrow i}$ is the decay branching ratio for the decay of the $j^{t h}$ to the $i^{t h}$ nuclide

In a transmutation environment (e.g., a neutron flux in a nuclear reactor) the time rate of change of the nuclide can be affected by other processes that can lead to the production and/or destruction of the $i^{t h}$ nuclide

$$
\frac{d N_{i}}{d t}=\sum_{j=1}^{J}\left(\lambda_{j} \beta_{j \rightarrow i}+\sum_{g=1}^{G} \sigma_{n, j}^{g}(t) \phi^{g}(t) \alpha_{j \rightarrow i}^{g}\right) N_{j}(t)-\left(\lambda_{i}+\sum_{g=1}^{G} \sigma_{a, i}^{g}(t) \phi^{g}(t)\right) N_{i}(t),
$$

where

$\sigma_{n, j}^{g}$ is the microscopic cross section for an interaction of type $n$ for the $j^{\text {th }}$ nuclide in group $g$

$\phi^{g}$ is the flux in group $g$

$\alpha_{j \rightarrow i}^{g}$ is the reaction branching ratio from the $j^{t h}$ to the $i^{t h}$ nuclide in group $g$

$\sigma_{a, i}^{g}$ is the microscopic absorption cross section for the $i^{t h}$ nuclide in group $g$

The problem can be formulated as a system of coupled ordinary differential equations with 
a properly defined initial condition,

$$
\begin{gathered}
\frac{d \vec{N}}{d t}=\boldsymbol{A}(t) \vec{N}, \\
\vec{N}(0)=\vec{N}_{0}
\end{gathered}
$$

The matrix coefficients take the form

$$
A_{i, j}=-\lambda_{i}^{e f f} \delta_{i j}+b_{i, j}^{e f f}
$$

where $\left(\delta_{i j}\right)$ is the Kronecker delta function and the effective decay/destruction coefficient, $\lambda_{i}^{e f f}$, populates the main diagonal of the matrix

$$
\lambda_{i}^{e f f}=\lambda_{i}+\sum_{g=1}^{G} \sigma_{a, i}^{g} \phi^{g},
$$

and the effective decay/transmutation coefficient, $b_{i, j}^{e f f}$, populates the off-diagonal terms

$$
b_{i, j}^{e f f}=\lambda_{j} \beta_{j \rightarrow i}+\sum_{g=1}^{G} \sigma_{n, j}^{g} \phi^{g} \alpha_{j \rightarrow i}^{g} .
$$




\section{The ISOXML Format}

An isotope decay and transmutation library is necessary to perform a variety of calculations related to nuclear processes (isotope depletion and transmutation, decay heat (energy deposition), fuel performance, radiotoxicity, etc.). The XML (eXtendable Markup Language) format has been chosen because of several appealing features, which include:

- It is in a general markup language for which robust parsers exist;

- Data is managed hierarchically with the natural XML tree structure;

- The sequence of nodes in an XML file on the same tree level is irrelevant, which gives great flexibility to manage all state points and all isotopes;

- It is both easily human and computer readable;

- It allows complete validity check;

- Comments can be inserted with the paired ${ }^{\prime}<!^{--^{\prime}}$ and ${ }^{\prime}-->^{\prime}$.

To save on storage, an XML library can be converted to a binary file. It worth noting that all symbols in a XML file are case sensitive.

\subsection{Terminology}

For clarity, we define several terms which will be used frequently in this document.

1. decay reaction - type of reactions due to radioactive decay. A list of valid decay reactions are included in Table 1

2. transmutation reaction - type of reactions initiated from neutron capture. A list of valid transmutation reactions are included in Table 2 . 
Table 1: Valid decay reaction types

\begin{tabular}{|c|c|c|}
\hline Identifier & Reaction & Description \\
\hline GenericDecay & Decay & generic decay (could be a chain of decays) \\
Betam & $\beta^{-}$ & electron production \\
Betap & $\beta^{+}$ & positron production or electron capture \\
DBetam & $2 \beta^{-}$ & double electron production \\
Alpha & $\alpha$ & alpha production \\
BetamAlpha & $\beta^{-} \alpha$ & electron and alpha production \\
IsomericTransition & $I T$ & isomeric transition \\
SpontaneousFission & $S F$ & spontaneous fission \\
Neutron & $\mathrm{N}$ & neutron production \\
DelayedNeutron & $\beta^{-} \mathrm{N}$ & electron and neutron production \\
\hline
\end{tabular}

Table 2: Valid neutron reaction types (consistent with the reaction types in the YAKXS multigroup library).

\begin{tabular}{|c|c|c|}
\hline Identifier & Reaction & Description \\
\hline NGamma & $(n, \gamma)$ & radiative capture \\
NAlpha & $(n, \alpha)$ & alpha production \\
N2Alpha & $(n, 2 \alpha)$ & 2 alpha production \\
N2N & $(n, 2 n)$ & 2 neutron production \\
N3N & $(n, 3 n)$ & 3 neutron production \\
N4N & $(n, 4 n)$ & 4 neutron production \\
NProton & $(n, p)$ & proton production \\
NNProton & $(n, n+p)$ & proton + neutron production \\
N2NProton & $(n, 2 n+p)$ & proton +2 neutron production \\
NDeuteron & $(n, d)$ & deuteron production \\
NTriton & $(n, t)$ & triton production \\
Fission & $(n, f)$ & fission \\
\hline
\end{tabular}




\subsection{Format}

The XML format of the decay and transmutation library is defined in this section. The name (XML tag) of the root element is always DecayTransmutationLibrary and its attributes are:

- Name

Description: the name of the library

Data type: string

Default value: N/A

Selection: any valid string

- $\mathrm{Ver}$

Description: the version of the decay library format

Data type: string

Default value: N/A

Selection: 1.0 is the only valid value.

- Generator

Description: the name of the generator of the library

Data type: string

Default value: 'INL'

Selection: any valid string.

The name (XML tag) of the subsequent element(s) is Isotope, which defines an isotope in the library. Its attributes are:

- Name

Description: the name of the isotope

Data type: string

Default value: empty string

Selection: any valid string 
- ZAID

Description: the ZAID number for this isotope based on ENDF-VI.

Data type: unsigned integer

Default value: 0

- DecayConstant

Description: the decay constant for this isotope in units of $\left[s^{-1}\right]$

Data type: real

Default value: 0.0

The valid XML tags under the Isotope tag include:

- DecayTypes

Description: list of non-fission decay reactions for this isotope

Data type: DecayType enum vector

Default value: empty vector

- DecayEnergies

Description: Recoverable energy for each non-fission decay reaction for this isotope in units of $[\mathrm{MeV}]$

Data type: real vector

Default value: empty vector

- DecayGammaEnergyFractions

Description: Fraction of recoverable decay energy released in the form of gamma rays for the decay reaction specified in DecayTypes

Data type: real vector

Default value: empty vector

- DecayBranchingRatios

Description: List of non-fission decay reaction branching ratios for this isotope

Data type: real vector

Default value: empty vector 
- DecayDaughters

Description: list of non-fission decay daughters generated by this isotope

Data type: string vector

Default value: empty vector

- NXTypes

Description: list of non-fission $(\mathrm{n}, \mathrm{x})$ reactions for this isotope

Data type: ReactionType enum vector

Default value: empty vector

- NXEnergies

Description: Recoverable energy for each non-fission $(\mathrm{n}, \mathrm{x})$ reaction for this isotope in units of $[\mathrm{MeV}]$

Data type: real vector

Default value: empty vector

- NXGammaEnergyFractions

Description: Fraction of recoverable non-fission $(\mathrm{n}, \mathrm{x})$ energy released in the form of gamma rays for the $(\mathrm{n}, \mathrm{x})$ reaction specified in NXTypes

Data type: real vector

Default value: empty vector

- NXBranchingRatios

Description: List of non-fission $(\mathrm{n}, \mathrm{x})$ reaction branching ratios for this isotope

Data type: real vector

Default value: empty vector

- NXDaughters

Description: list of non-fission $(\mathrm{n}, \mathrm{x})$ daughters generated by this isotope

Data type: string vector

Default value: empty vector

- FissionData

The fission data is treated separately. 
The valid XML tags under the FissionData tag include:

- NeutronInduced

Description: contain the data from neutron induced reactions.

- Spontaneous

Description: describe the data from spontaneous fission events.

The valid XML tags under the NeutronInduced tag include:

\section{- IncidentNeutronEnergy}

Description: list neutron energies in $\mathrm{MeV}$ for the neutron induced fission reaction tabulations. A value of 0 indicates that the isotope is capable of undergoing neutroninduced fission, but the incident neutron energy and subsequent fission product yields are not known.

Data type: real vector

Default value: empty vector

- FissionTable

Description: contains the data tables from neutron induced fission events for an incident neutron energy.

The FissionTable tag includes the following attributes:

- GridIndex

Description: grid value corresponding to the incident neutron energy entry in the IncidentNeutronEnergy tag.

Data type: integer

Default value: N/A 


\section{- TotalFissionEnergy}

Description: Total recoverable energy in units of $[\mathrm{MeV}]$ for the fission reaction of this isotope and subsequent decay of fission products corresponding to the incident neutron energy entry in the IncidentNeutronEnergy tag.

Data type: real

Default value: N/A

- PromptFissionEnergy

Description: Prompt recoverable energy in $[\mathrm{MeV}]$ for the fission reaction of this isotope corresponding to the incident neutron energy entry in the IncidentNeutronEnergy tag.

Data type: real

Default value: N/A

\section{- PromptFissionGammaEnergyFraction}

Description: Fraction of PromptFissionEnergy released as gamma rays corresponding to the incident neutron energy entry in the IncidentNeutronEnergy tag.

Data type: real

Default value: N/A

The FissionTable tag includes the following tags:

\section{- YieldFractions}

Description: list of yield fractions for the various fission products corresponding to the incident neutron energy entry in the IncidentNeutronEnergy tag.

Data type: real vector

Default value: empty vector

- FissionProducts

Description: list of fission products for fission reactions corresponding to the incident neutron energy entry in the IncidentNeutronEnergy tag.

Data type: string vector

Default value: empty vector 
The Spontaneous tag includes the following attributes:

- TotalSFEnergy

Description: Total recoverable energy in units of $[\mathrm{MeV}]$ for the spontaneous fission of this isotope and subsequent decay reactions of fission products.

Data type: real

Default value: 0.0

- PromptSFEnergy

Description: Prompt recoverable energy in units of $[\mathrm{MeV}]$ for the spontaneous fission of this isotope.

Data type: real

Default value: 0.0

- PromptSFGammaEnergyFraction

Description: Fraction of prompt recoverable energy released in the form of gamma rays for the spontaneous fission of this isotope.

Data type: real

Default value: 0.0

- BranchingRatio

Description: Branching ratio for spontaneous fission of this isotope.

Data type: real

Default value: 0.0

The Spontaneous tag includes the following tags:

- YieldFractions

Description: list of yield fractions for the various spontaneous fission products.

Data type: real vector

Default value: empty vector 


\section{- SFProducts}

Description: list of spontaneous fission products.

Data type: string vector

Default value: empty vector

\subsection{A Sample Valid Library}

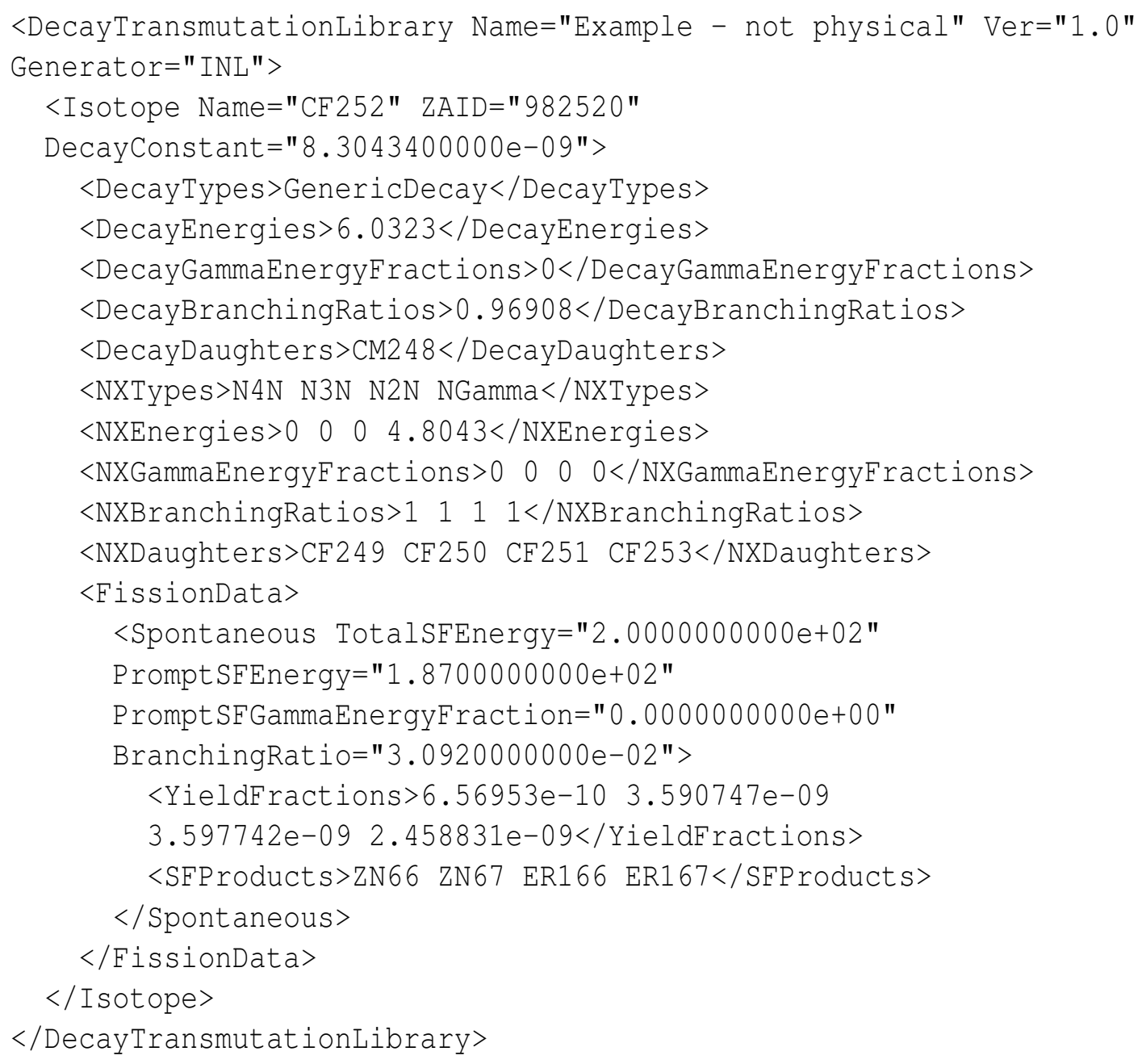




\section{Data Loader}

Data loaders were implemented in MAMMOTH for reading decay data files in the ISOXML format and for reading ENDF format decay data files in ORIGEN format. The ORIGEN data set includes over 1,600 unique isotopes and satisfies the needs of the vast majority of transmutation problems. ORIGEN also sets the lower limit of isotopes' half-lives to 1 millisecond to avoid numerical problems arising from the separation of time-scales that are present in the transmutation problem. This is a reasonable simplification for most realworld quasi-steady-state depletion problems, whose characteristic time scale will likely never be below the order of seconds. In contrast, transient problems may have relevant time scales on the order of microseconds or shorter.

ORIGEN has two data files it uses for determining the results of radioactive decay and the products of fission events (outlined in the SCALE 6.2 Manual - Section 5.2.1):

- ENDF/B-VII.1 Decay Data File [origen.rev03.decay.data]

- ENDF/B-VII.0 Based Fission Yield Data File [origen.rev05.yields.data]

The ORIGEN ENDF/B-VII.1 decay data file includes the following:

- Nuclide Identifier (ZAID)

- Half-Life

- Beta Decay to Ground State Daughter Fraction

- Beta Decay to Metastable Daughter Fraction

- Positron Decay or Electron Capture to Ground State Daughter Fraction

- Positron Decay or Electron Capture to Metastable Daughter Fraction

- Alpha Particle Decay Fraction

- Isomeric Transition Decay Fraction

- Spontaneous Fission Decay Fraction 
- Delayed Neutron Decay (Beta Particle + Neutron) Fraction

- Double Beta Decay Fraction

- Neutron Decay Fraction

- Combined Beta Minus decay and alpha particle emission

- Fraction of Recoverable Decay Energy associated with Gamma Rays

- Recoverable Energy per Decay (in MeV)

- Natural atom isotopic abundance (in percent)

- Maximum Permissible Concentration in Air

- Maximum Permissible Concentration in Water

The fission yield data file has fission product yield data at incident neutron energies as outlined in Table 3. For incident neutron energies between a pair of tabulated values, ORIGEN uses linear interpolation to obtain the fission product yield. For neutron energies outside of the tabulated range, the closest relevant value of the fission product yield is selected. This interpolation functionality will subsequently be added to MAMMOTH at a later time and was not part of this work scope.

ORIGEN also has the energies for fission events and neutron capture events for selected nuclides. This information is hard-coded into ORIGEN (there is no data file for it) so the information is also hard-coded into the data loader. Recoverable neutron capture and neutron-induced fission energies for a variety of actinides are shown in Table 4 and recoverable neutron interaction energies for other nuclei of interest are shown in Table 5 .

The data loader thus can read the same data files that ORIGEN uses in order to allow MAMMOTH to use said data in depletion problems or output the ORIGEN decay data in the ISOXML format. The cross-section files ORIGEN utilizes do not follow the same format and thus a different data loader was implemented which can read the default crosssection and decay libraries provided by the ORIGEN-ARP module in SCALE 6.1.

The data loaders are implemented as member functions of MAMMOTH's DecayTransmutationLibrary class. 
Table 3: Nuclides with Fission Product Branching Ratios at Given Neutron Energies

\begin{tabular}{|c|c|c|c|}
\hline Nuclide & 0.0253 [eV] & 2 [MeV] & 14 [MeV] \\
\hline Th-227 & $\checkmark$ & & \\
Th-229 & $\checkmark$ & $\checkmark$ & $\checkmark$ \\
Th-232 & & $\checkmark$ & \\
Pa-231 & & & \\
U-232 & $\checkmark$ & $\checkmark$ & $\checkmark$ \\
U-233 & $\checkmark$ & $\checkmark$ & $\checkmark$ \\
U-234 & & $\checkmark$ & $\checkmark$ \\
U-235 & $\checkmark$ & $\checkmark$ & $\checkmark$ \\
U-236 & & $\checkmark$ & \\
U-237 & & $\checkmark$ & $\checkmark$ \\
U-238 & & $\checkmark$ & $\checkmark$ \\
Np-237 & $\checkmark$ & $\checkmark$ & \\
Np-238 & & $\checkmark$ & \\
Pu-238 & & $\checkmark$ & $\checkmark$ \\
Pu-239 & $\checkmark$ & $\checkmark$ & $\checkmark$ \\
Pu-240 & $\checkmark$ & $\checkmark$ & \\
Pu-241 & $\checkmark$ & $\checkmark$ & $\checkmark$ \\
Pu-242 & $\checkmark$ & $\checkmark$ & $\checkmark$ \\
Am-241 & $\checkmark$ & $\checkmark$ & \\
Am-242m & $\checkmark$ & $\checkmark$ & \\
Am-243 & & $\checkmark$ & \\
Cm-242 & & $\checkmark$ & \\
Cm-243 & $\checkmark$ & $\checkmark$ & \\
Cm-244 & & $\checkmark$ & \\
Cm-245 & $\checkmark$ & & \\
Cm-246 & & & \\
Cm-248 & & $\checkmark$ & \\
Cf-249 & $\checkmark$ & & \\
Cf-251 & $\checkmark$ & \\
Es-254 & $\checkmark$ & & \\
\hline
\end{tabular}


Table 4: Recoverable Energy for Actinides [MeV]

\begin{tabular}{|c|c|c|c|}
\hline Nuclide & Fission Energy & Capture Energy & Capture Reaction \\
\hline Th-230 & 190.00 & 5.010 & $(\mathrm{n}, \gamma)$ \\
Th-232 & 189.21 & 4.786 & $(\mathrm{n}, \gamma)$ \\
Th-233 & 190.00 & 6.080 & $(\mathrm{n}, \gamma)$ \\
$\mathrm{Pa}-231$ & 190.00 & 5.660 & $(\mathrm{n}, \gamma)$ \\
$\mathrm{Pa}-233$ & 189.10 & 5.197 & $(\mathrm{n}, \gamma)$ \\
$\mathrm{U}-232$ & 200.00 & 5.930 & $(\mathrm{n}, \gamma)$ \\
$\mathrm{U}-233$ & 191.29 & 6.841 & $(\mathrm{n}, \gamma)$ \\
$\mathrm{U}-234$ & 190.30 & 5.297 & $(\mathrm{n}, \gamma)$ \\
$\mathrm{U}-235$ & 194.02 & 6.545 & $(\mathrm{n}, \gamma)$ \\
$\mathrm{U}-236$ & 192.80 & 5.124 & $(\mathrm{n}, \gamma)$ \\
$\mathrm{U}-238$ & 198.12 & 4.804 & $(\mathrm{n}, \gamma)$ \\
$\mathrm{Np}-237$ & 195.10 & 5.490 & $(\mathrm{n}, \gamma)$ \\
$\mathrm{Np}-239$ & 200.00 & 4.970 & $(\mathrm{n}, \gamma)$ \\
$\mathrm{Pu}-238$ & 197.80 & 5.550 & $(\mathrm{n}, \gamma)$ \\
$\mathrm{Pu}-239$ & 200.05 & 6.533 & $(\mathrm{n}, \gamma)$ \\
$\mathrm{Pu}-240$ & 199.79 & 5.241 & $(\mathrm{n}, \gamma)$ \\
$\mathrm{Pu}-241$ & 202.22 & 6.301 & $(\mathrm{n}, \gamma)$ \\
$\mathrm{Pu}-242$ & 200.62 & 5.071 & $(\mathrm{n}, \gamma)$ \\
$\mathrm{Pu}-243$ & 200.00 & 6.020 & $(\mathrm{n}, \gamma)$ \\
$\mathrm{Am}-241$ & 202.30 & 5.529 & $(\mathrm{n}, \gamma)$ \\
$\mathrm{Am}-242 \mathrm{~m}$ & 202.29 & 6.426 & $(\mathrm{n}, \gamma)$ \\
$\mathrm{Am}-243$ & 202.10 & 5.363 & $(\mathrm{n}, \gamma)$ \\
$\mathrm{Cm}-244$ & 200.00 & 6.451 & $(\mathrm{n}, \gamma)$ \\
$\mathrm{Cm}-245$ & 200.00 & 6.110 & $(\mathrm{n}, \gamma)$ \\
\hline
\end{tabular}


Table 5: Recoverable energy $[\mathrm{MeV}]$ values for Non-Actinide Neutron Activation

\begin{tabular}{|c|c|c|}
\hline Nuclide & Capture Energy & Reaction \\
\hline $\mathrm{H}-1$ & 2.225 & $(\mathrm{n}, \gamma)$ \\
\hline B-10 & 2.790 & $(\mathrm{n}, \alpha)$ \\
\hline $\mathrm{O}-16$ & 4.143 & $(\mathrm{n}, \gamma)$ \\
\hline $\mathrm{Fe}-56$ & 7.600 & $(\mathrm{n}, \gamma)$ \\
\hline $\mathrm{Ni}-58$ & 9.020 & $(\mathrm{n}, \gamma)$ \\
\hline Zr-90 & 7.203 & $(\mathrm{n}, \gamma)$ \\
\hline Zr-91 & 8.635 & $(\mathrm{n}, \gamma)$ \\
\hline Zr-92 & 6.758 & $(\mathrm{n}, \gamma)$ \\
\hline Zr-96 & 5.571 & $(\mathrm{n}, \gamma)$ \\
\hline Мo-95 & 9.154 & $(\mathrm{n}, \gamma)$ \\
\hline Tc-95 & 7.710 & $(\mathrm{n}, \gamma)$ \\
\hline Ru-101 & 9.216 & $(\mathrm{n}, \gamma)$ \\
\hline Rh-103 & 6.999 & $(\mathrm{n}, \gamma)$ \\
\hline Rh-105 & 7.094 & $(\mathrm{n}, \gamma)$ \\
\hline Ag-109 & 6.825 & $(\mathrm{n}, \gamma)$ \\
\hline Xe-131 & 8.936 & $(\mathrm{n}, \gamma)$ \\
\hline Xe-135 & 7.880 & $(\mathrm{n}, \gamma)$ \\
\hline Cs-133 & 6.704 & $(\mathrm{n}, \gamma)$ \\
\hline Cs-134 & 6.550 & $(\mathrm{n}, \gamma)$ \\
\hline Nd-143 & 7.817 & $(\mathrm{n}, \gamma)$ \\
\hline Nd-145 & 7.565 & $(\mathrm{n}, \gamma)$ \\
\hline Pm-147 & 5.900 & $(\mathrm{n}, \gamma)$ \\
\hline Pm-148 & 7.266 & $(\mathrm{n}, \gamma)$ \\
\hline Pm-148m & 7.266 & $(\mathrm{n}, \gamma)$ \\
\hline Sm-147 & 8.140 & $(\mathrm{n}, \gamma)$ \\
\hline Sm-149 & 7.982 & $(\mathrm{n}, \gamma)$ \\
\hline Sm-150 & 5.596 & $(\mathrm{n}, \gamma)$ \\
\hline Sm-151 & 8.258 & $(\mathrm{n}, \gamma)$ \\
\hline Sm-152 & 5.867 & $(\mathrm{n}, \gamma)$ \\
\hline Eu-153 & 6.444 & $(\mathrm{n}, \gamma)$ \\
\hline Eu-154 & 8.167 & $(\mathrm{n}, \gamma)$ \\
\hline Eu-155 & 6.490 & $(\mathrm{n}, \gamma)$ \\
\hline
\end{tabular}




\section{Architecture}

The architecture improvements to MAMMOTH primarily focus on the addition of a new MOOSE Depletion User Object (UO) and updates to the existing Depletion Material.

The new Depletion UO functionality includes the capability to:

- create and hold the master decay and transmutation library, which contains all of the decay and transmutation data from the input decay data file,

- create working decay and transmutation libraries, which contain data on all isotopes relevant for a given depletion zone (or ID) and constructs the depletion matrix for said zone when provided cross section data,

- store the list of isotopes being tracked and the number density $(\vec{N})$ of each isotope in each depletion zone,

- update the number densities after each time step, and

- solve the depletion matrix using CRAM.

The Depletion Material functionality includes:

- loading and updating the cross-sections and reaction rates of each isotope and

- using the isotopic number densities given by the Depletion UO to generate macroscopic cross sections for use in the neutron transport calculation.

The user is given the ability to specify a scalar flux level to deplete the system with, in which case the flow of data and calculations is shown in Figure 1 . Alternatively, the user may specify a power level to deplete the system with, in which case the calculations proceed largely the same as with a user-specified scalar flux with the exception that the flux is evaluated by a transport solver and is scaled in order to ensure that the desired power level is being achieved. This extra step can be seen in Figure 2 .

Regardless of which option is selected, the process of microscopic depletion proceeds as follows. The decay data files are directly loaded into the Depletion UO to form the master DecayTransmutationLibrary, whereas the cross section data is loaded by the Depletion Material into a MultigroupLibrary class, which is then processed into a mixing table 
(MixingTable), that holds microscopic cross sections of all isotopes at a particular state point, and passed to the Depletion UO. The Depletion UO generates a working decay and transmutation library (WorkingDecayTransmutationLibrary) for each individual depletion zone, which depends on the isotope composition in the respective depletion zone. The WorkingDecayTransmutationLibrary accepts the cross section data from the Depletion UO and generates the decay and transmutation matrices. Then the decay and transmutation matrices are summed to produce depletion matrix, for a given depletion zone. The depletion matrix and isotopic number densities from the previous time step are then passed to the CRAM solver, which is executed by the Depletion UO and calculates the resulting isotopic number densities for the end of the current time step. The new number densities are then sent to the Depletion Material so they can be used in the cross section mixer to develop macroscopic cross sections for the neutron transport calculation.

If the user specifies a power level for the system, which may be time-dependent, then an extra step occurs where the raw neutron flux, either specified by the user or the result of a neutron transport calculation, is scaled to the "true" neutron flux level. This calculation is performed by using the Depletion UO to calculate the total decay power from the system (which can also be used to calculate the decay power density for a given depletion zone) which is then subtracted from the rated power level to determine the total power expected from prompt reactions as a result of neutron irradiation (e.g., fission, radiative capture), referred to as the prompt power. In the event that total decay power exceeds the userspecified power level an error is thrown. The raw prompt power density can be calculated with the raw neutron flux and the reaction cross sections stored by the Depletion Material, with the number densities from the Depletion UO. This raw prompt power density can be used to calculate the total raw prompt power in the system which, when can be used to calculate the factor the raw neutron flux must be scaled by in order for the total power of the depletion calculation to equal the user-specified power level. 


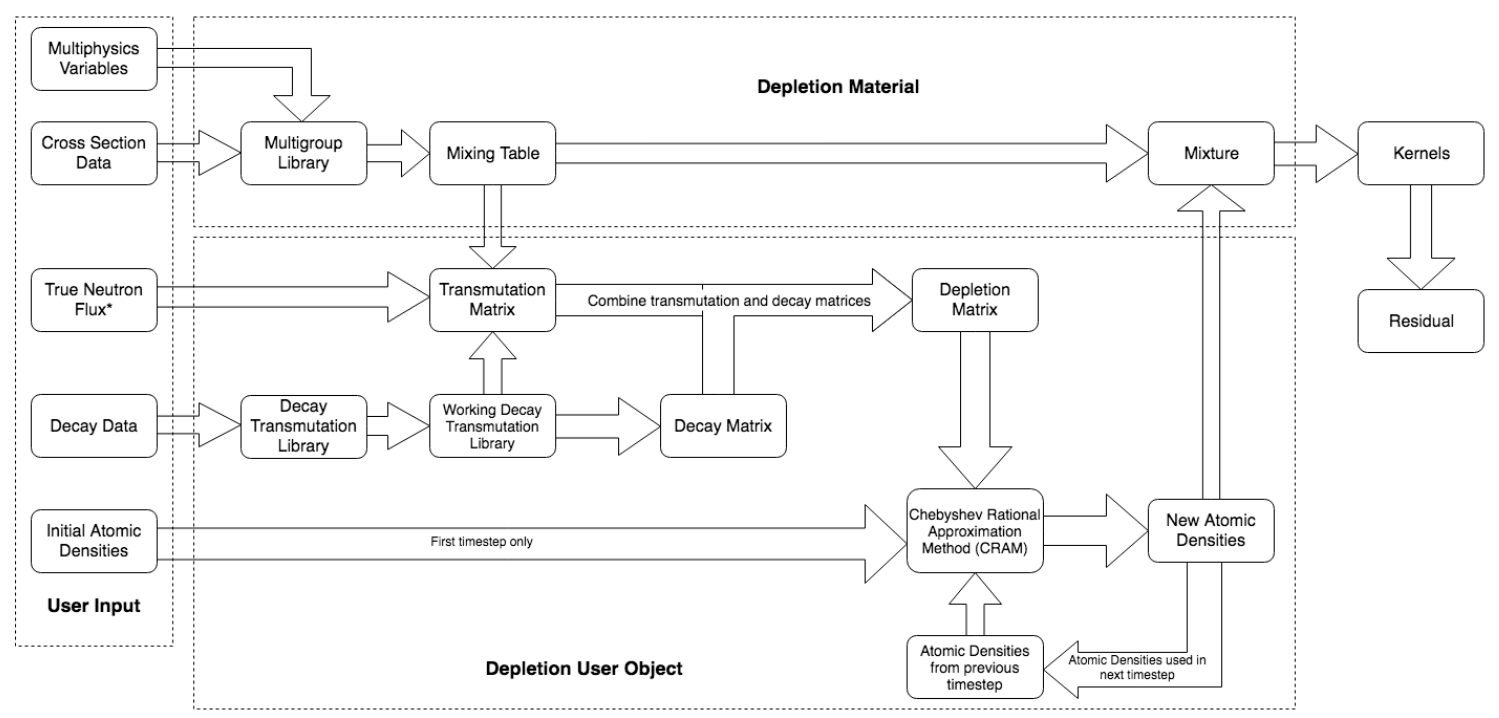

Figure 1: Flowchart of data transfer when performing microscopic depletion calculations. Note that the neutron flux can be specified by the user or taken from a neutron transport calculation. 


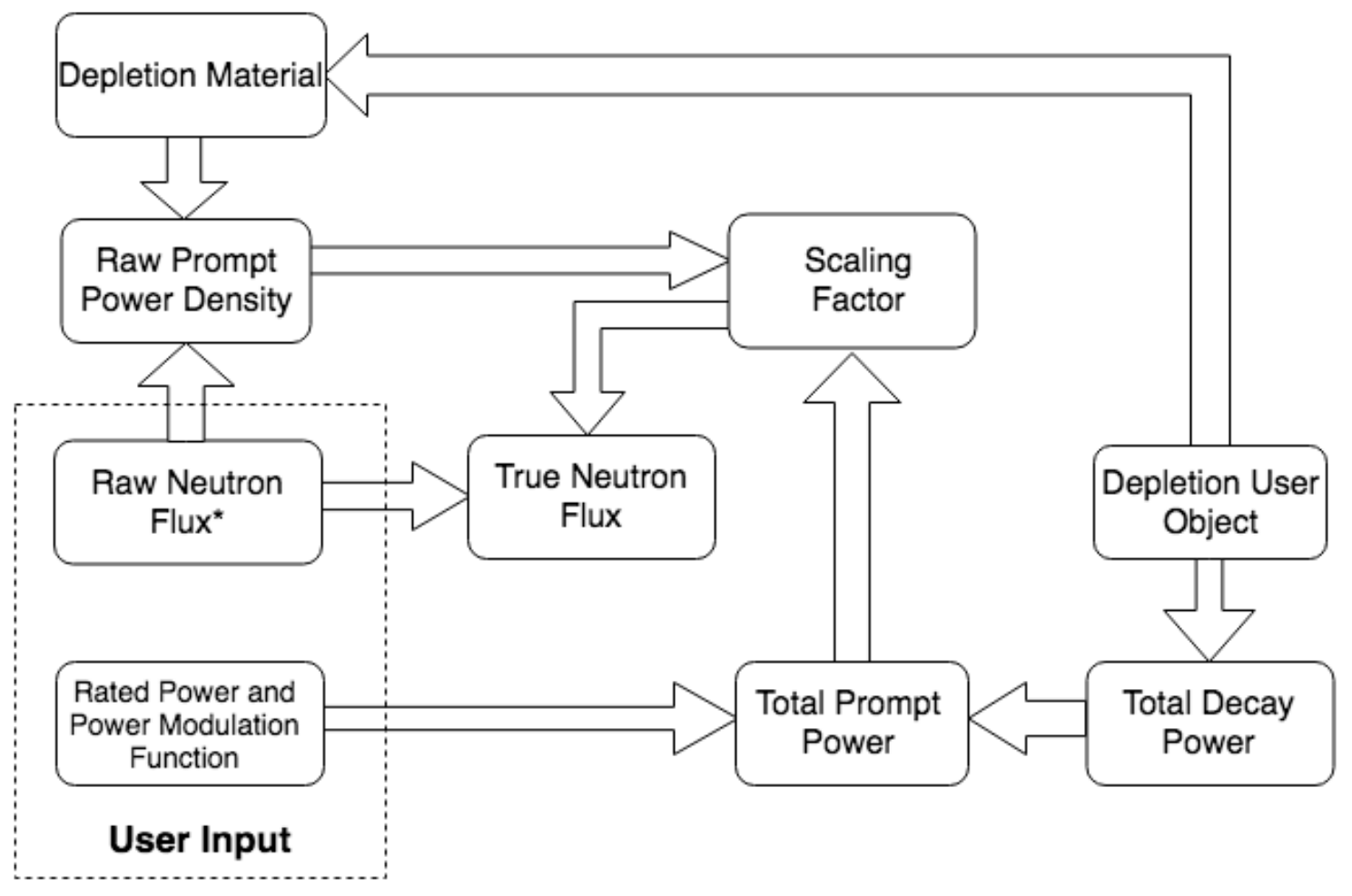

Figure 2: Flowchart of simplified data transfer when performing microscopic depletion calculations with a constant power level. Note that the neutron flux can be specified by the user or taken from a neutron transport calculation. 


\section{Test Problems}

The test problems described in this section are used to verify the changes to the microscopic depletion implementation in MAMMOTH and are based on the initial work documented in [9]. The level of complexity of each exercise is increased at each step to provide a comprehensive approach to benchmarking the implementation in MAMMOTH. Two analytic solutions are included in Sections 6.1 and 6.2. Comparisons between MAMMOTH and the depletion codes DRAGON5 and ORIGEN are also shown in Sections 6.3 and 6.4, respectively. The results to these problems are provided in Section 7. A CRAM order of 16 was used for all tests in MAMMOTH.

\subsection{Thorium Series}

The thorium-232 decay series, shown in Figure 3][11], provides a very good data set for benchmarking the decay of isotopes, since it includes a variety of half-lives from billions of years to $10^{-7}$ seconds. A converged accelerated semi-analytical benchmark for this series is available for comparison [12].

The benchmark dataset is provided in Tables 6 through 8 . The solution provided in Table 8 was obtained with the Doubling algorithm [12] where the time steps are found adaptively with Richardson or Wynn-Epsilon extrapolation. These results were confirmed via a Transmutation Trajectory Analysis (TTA) solution. 


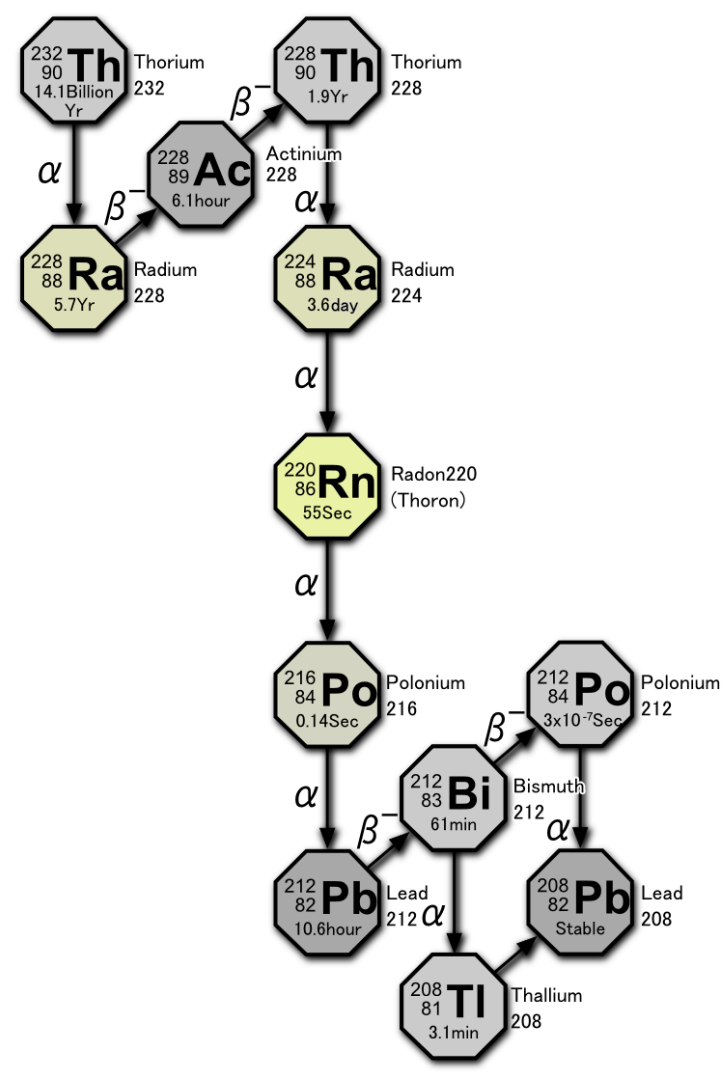

Figure 3: Thorium-232 decay series. 
Table 6: Decay constants for the Thorium Series (per day)

\begin{tabular}{c|c} 
Isotope & Decay Constant \\
\hline Th-232 & $1.351625 \mathrm{E}-13$ \\
Ra-228 & $3.302667 \mathrm{E}-04$ \\
Ac-228 & $2.661685 \mathrm{E}+00$ \\
Th-228 & $9.934261 \mathrm{E}-04$ \\
$\mathrm{Ra}-224$ & $1.908497 \mathrm{E}-01$ \\
$\mathrm{Rn}-220$ & $1.077121 \mathrm{E}+03$ \\
$\mathrm{Po}-216$ & $4.130201 \mathrm{E}+05$ \\
$\mathrm{~Pb}-212$ & $1.563490 \mathrm{E}+00$ \\
$\mathrm{Bi}-212$ & $1.648442 \mathrm{E}+01$ \\
$\mathrm{Po}-212$ & $2.002940 \mathrm{E}+11$ \\
$\mathrm{Tl}-208$ & $3.269348 \mathrm{E}+02$ \\
\hline
\end{tabular}

Table 7: Branching ratios for $\mathrm{Bi}-212$

\begin{tabular}{c|c} 
Daughter Isotope & Branching Ratio \\
\hline Po-212 & 0.6406 \\
Tl-208 & 0.3594
\end{tabular}


Table 8: Reference solution number densities for isotopes in the thorium-232 series after 1 year. $\left(1 \times 10^{-12}\right.$ relative error)

\begin{tabular}{|c|c|c|c|c|}
\hline Time (days) & Th-232 & Ra-228 & Ac-228 & Th-228 \\
\hline 36.5 & $6.022999999970 \mathrm{E}+23$ & $2.953567638154 \mathrm{E}+12$ & $3.627339589142 \mathrm{E}+08$ & $1.726783939692 \mathrm{E}+10$ \\
\hline 73.0 & $6.022999999941 \mathrm{E}+23$ & $5.871744543953 \mathrm{E}+12$ & 7.248715742555E+08 & $6.867693005511 \mathrm{E}+10$ \\
\hline 109.5 & $6.022999999911 \mathrm{E}+23$ & $8.754954782151 E+12$ & $1.082669923668 \mathrm{E}+09$ & $1.525986405848 \mathrm{E}+11$ \\
\hline 146.0 & $6.022999999881 \mathrm{E}+23$ & $1.160361733620 \mathrm{E}+13$ & $1.436181001830 \mathrm{E}+09$ & $2.674636649886 \mathrm{E}+11$ \\
\hline 182.5 & $6.022999999851 \mathrm{E}+23$ & $1.441814616915 \mathrm{E}+13$ & $1.785456180401 \mathrm{E}+09$ & 4.117635120055E+11 \\
\hline 219.0 & $6.022999999822 \mathrm{E}+23$ & $1.719895028378 \mathrm{E}+13$ & $2.130546215485 \mathrm{E}+09$ & $5.840482803810 \mathrm{E}+11$ \\
\hline 255.5 & $6.022999999792 \mathrm{E}+23$ & $1.994643378205 \mathrm{E}+13$ & $2.471501255008 \mathrm{E}+09$ & $7.829245140706 \mathrm{E}+11$ \\
\hline 292.0 & $6.022999999762 \mathrm{E}+23$ & $2.266099592383 \mathrm{E}+13$ & $2.808370846007 \mathrm{E}+09$ & $1.007053134518 \mathrm{E}+12$ \\
\hline 328.5 & $6.022999999733 \mathrm{E}+23$ & $2.534303118491 E+13$ & 3.141203941827E+09 & $1.255147447262 \mathrm{E}+12$ \\
\hline 365.0 & $6.022999999703 \mathrm{E}+23$ & $2.799292931431 \mathrm{E}+13$ & $3.470048909235 \mathrm{E}+09$ & $1.525971220201 \mathrm{E}+12$ \\
\hline Time (day & $\mathrm{Ra}-224$ & Rn-220 & Po-216 & $\mathrm{Pb}-212$ \\
\hline 36.5 & $6.770295695164 \mathrm{E}+07$ & $1.199525306804 \mathrm{E}+04$ & $3.128258654688 \mathrm{E}+01$ & 7.939993206442E+06 \\
\hline 73.0 & $3.102328977782 \mathrm{E}+08$ & $5.496713712636 \mathrm{E}+04$ & $1.433495695291 \mathrm{E}+02$ & $3.717037958899 \mathrm{E}+07$ \\
\hline 109.5 & 7.231989443712E+08 & $1.281377826921 \mathrm{E}+05$ & $3.341723329185 \mathrm{E}+02$ & $8.722319214702 \mathrm{E}+07$ \\
\hline 146.0 & 1.29838979 & $2.300522691959 \mathrm{E}+05$ & $5.999565686358 \mathrm{E}+02$ & $1.570954782267 \mathrm{E}+08$ \\
\hline 182.5 & 2.02790849 & $3.593113727562 \mathrm{E}+05$ & $9.370532207864 \mathrm{E}+02$ & $2.458226146684 \mathrm{E}+08$ \\
\hline 219.0 & 2.90416470 & $5.145701970348 \mathrm{E}+05$ & $1.341954915050 \mathrm{E}+03$ & $3.524774300961 E+08$ \\
\hline 255.5 & $1 \mathrm{E}+09$ & $6.945361853018 \mathrm{E}+05$ & $6 \mathrm{E}+03$ & $4.761688345707 \mathrm{E}+08$ \\
\hline & 5.06799442 & 8.979672033 & $2.341821418979 \mathrm{E}+03$ & $6.160404979930 \mathrm{E}+08$ \\
\hline 328.5 & $6.341821336098 \mathrm{E}+09$ & $1.123669691062 \mathrm{E}+06$ & $2.930434146939 \mathrm{E}+03$ & $7.712695760090 \mathrm{E}+08$ \\
\hline 365.0 & $7.734872062336 \mathrm{E}+09$ & $1.370496881064 \mathrm{E}+06$ & $3.574138286889 \mathrm{E}+03$ & $9.410654817207 \mathrm{E}+08$ \\
\hline Time (days) & Bi-212 & Po-212 & Tl-208 & $\mathrm{Pb}-208$ \\
\hline 36.5 & $7.501737681487 \mathrm{E}+05$ & $3.955073325537 \mathrm{E}-05$ & $1.359150693723 \mathrm{E}+04$ & $1.310116157156 \mathrm{E}+08$ \\
\hline & $3.519211116439 \mathrm{E}+06$ & $1.855401855481 \mathrm{E}-04$ & $6.376713399337 \mathrm{E}+04$ & $1.313896772178 \mathrm{E}+09$ \\
\hline & $8.263344020327 \mathrm{E}+06$ & $4.356608148962 \mathrm{E}-04$ & $1.497343094422 \mathrm{E}+05$ & 4.761869212390E+09 \\
\hline & $1.488744026561 \mathrm{E}+07$ & 7.848970515905E-04 & $2.697691038058 \mathrm{E}+05$ & $1.163451205090 \mathrm{E}+10$ \\
\hline & $2.330000268061 \mathrm{E}+07$ & $1.228424972983 \mathrm{E}-03$ & $4.222134591463 \mathrm{E}+05$ & $2.303525827430 \mathrm{E}+10$ \\
\hline 219.0 & $3.341308648481 \mathrm{E}+07$ & $1.761607945931 \mathrm{E}-03$ & $6.054736918563 \mathrm{E}+05$ & $4.001356399748 \mathrm{E}+10$ \\
\hline & $4.514216930889 \mathrm{E}+07$ & 2.379989773984E-03 & $8.180181372398 \mathrm{E}+05$ & $6.356700654227 \mathrm{E}+10$ \\
\hline 292.0 & $5.840602583819 \mathrm{E}+07$ & 3.079288088324E-03 & $1.058374877876 \mathrm{E}+06$ & $9.464330569818 \mathrm{E}+10$ \\
\hline 328.5 & $7.312660696192 \mathrm{E}+07$ & $3.855387976254 \mathrm{E}-03$ & $1.325129553631 \mathrm{E}+06$ & $1.341422709222 \mathrm{E}+11$ \\
\hline 365.0 & $8.922892326710 \mathrm{E}+07$ & 4.704335838763E-03 & $1.616923250406 \mathrm{E}+06$ & $1.829176771398 \mathrm{E}+11$ \\
\hline
\end{tabular}

\subsubsection{MAMMOTH-to-ORIGEN Thorium Series Comparison}

The results for the thorium series decay in MAMMOTH are compared to the results from ORIGEN (Oak Ridge Isotope Generation code). ORIGEN was run with a CRAM approximation order of 16, the same as MAMMOTH. The same dataset used by ORIGEN was also used by MAMMOTH for the purposes of comparison as the decay data for ORIGEN 
differs from the previous benchmark. Instead of decay constants, data is stored in terms of half-lives, which are shown in Table 9. It should be noted that ORIGEN assumes no half-life is shorter than one millisecond, which results in polonium-212 having a much longer half-life for the purposes of this comparison than it does in reality. The bismuth-212 branching ratios for ORIGEN are the same as those in the benchmark as outlined in Table 7.

Table 9: Half-Lives for the Thorium Series

\begin{tabular}{c|c} 
Isotope & Half-Life \\
\hline Th-232 & $1.4050 \mathrm{E}+10[\mathrm{y}]$ \\
$\mathrm{Ra}-228$ & $5.7500 \mathrm{E}+00[\mathrm{y}]$ \\
Ac-228 & $6.1500 \mathrm{E}+00[\mathrm{~h}]$ \\
Th-228 & $1.9120 \mathrm{E}+00[\mathrm{y}]$ \\
$\mathrm{Ra}-224$ & $3.6600 \mathrm{E}+00[\mathrm{~d}]$ \\
$\mathrm{Rn}-220$ & $5.5600 \mathrm{E}+01[\mathrm{~s}]$ \\
$\mathrm{Po}-216$ & $1.4500 \mathrm{E}-01[\mathrm{~s}]$ \\
$\mathrm{Pb}-212$ & $1.0640 \mathrm{E}+01[\mathrm{~h}]$ \\
$\mathrm{Bi}-212$ & $1.0092 \mathrm{E}+00[\mathrm{~h}]$ \\
$\mathrm{Po}-212$ & $1.0000 \mathrm{E}-03[\mathrm{~s}]$ \\
$\mathrm{Tl}-208$ & $3.0530 \mathrm{E}+00[\mathrm{~m}]$ \\
\hline
\end{tabular}

\subsection{Constant Flux in a Homogeneous System}

The next step is to check the refactored microscopic depletion implementation against a constant flux analytic solution. For this, the simple uranium-235, iodine-135, and xenon135 transmutation chain is employed. The analytic solution consists of a constant flux irradiation for 100 hours followed by decay (zero flux) for another 100 hours, as shown in Figure 4. 


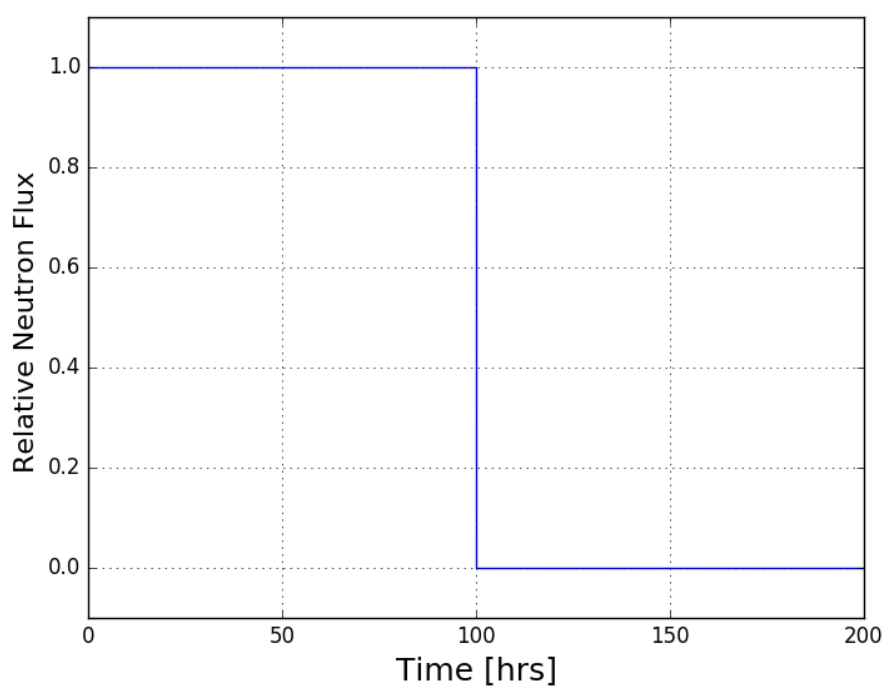

Figure 4: Irradiation history for constant flux test.

\subsection{MAMMOTH-to-DRAGON5}

In order to demonstrate the correctness of the depletion implementation in MAMMOTH, a comparison between DRAGON5 [13], a depletion application maintained by the École Polytechnique de Montréal, and MAMMOTH was performed. The reference DRAGON5 problem was a homogenized pressurized water reactor fuel cell exposed to a constant flux of $8 \times 10^{14}\left[\frac{n}{\mathrm{~cm}^{2} * s}\right]$ for 600 days. One energy group cross sections as well as decay and transmutation data were extracted from DRAGON5 for 297 isotopes. The significance of running systems of hundreds of isotopes is the inability to achieve a true analytic solution with a reasonable amount of time and effort to be practical. As a result, approximation methods are a necessity when testing real-world reactor conditions thus the need for such tests.

\subsection{MAMMOTH-to-ORIGEN Fuel Pin Comparison}

In order to test the constant power depletion capabilities of MAMMOTH, a comparison was made against ORIGEN using the Westinghouse $17 \times 17$ assembly with $5 \%$ enrichment 
library (w17_e50.arplib), processed by the ORIGEN-ARP module in SCALE 6.1. From this library decay, transmutation, and one energy group cross section data was loaded for 1,400 isotopes. As ORIGEN stores cross sections for various burnup levels, the $16.5 \mathrm{GWd}$ burnup cross-sections were used for the entirety of both the ORIGEN and MAMMOTH calculations. The system was run at a constant power level of $30 \mathrm{MW}$ for 1100 days with a total of 62 timesteps performed. Increasing the number of isotopes increased the runtime, as expected, while also demonstrating the ability of MAMMOTH to fully duplicate the functionality of ORIGEN as 1,400 isotope systems are presently impossible to solve analytically and many approximation methods that are capable of performing depletion for a few hundred isotopes are unable to run 1,400 isotope systems. ORIGEN was running using its default matrix exponential approximation method (referred to in the manual as MATREX) as CRAM was not available as a solution method for ORIGEN in SCALE 6.1. 


\section{Results}

The results to the test problems introduced in Section 6 are discussed in this section and follow the same order.

\subsection{Thorium Series}

The solution to the thorium approaches the precision of the reference calculation, as shown in Table 10. The greatest deviation from the reference solution occurs for the concentration of $\mathrm{Pb}-208$, which is the stable isotope in the thorium series. However, these differences can be neglected because the differences are extremely small and very close to the precision of the reference, as shown in Figure 5 .

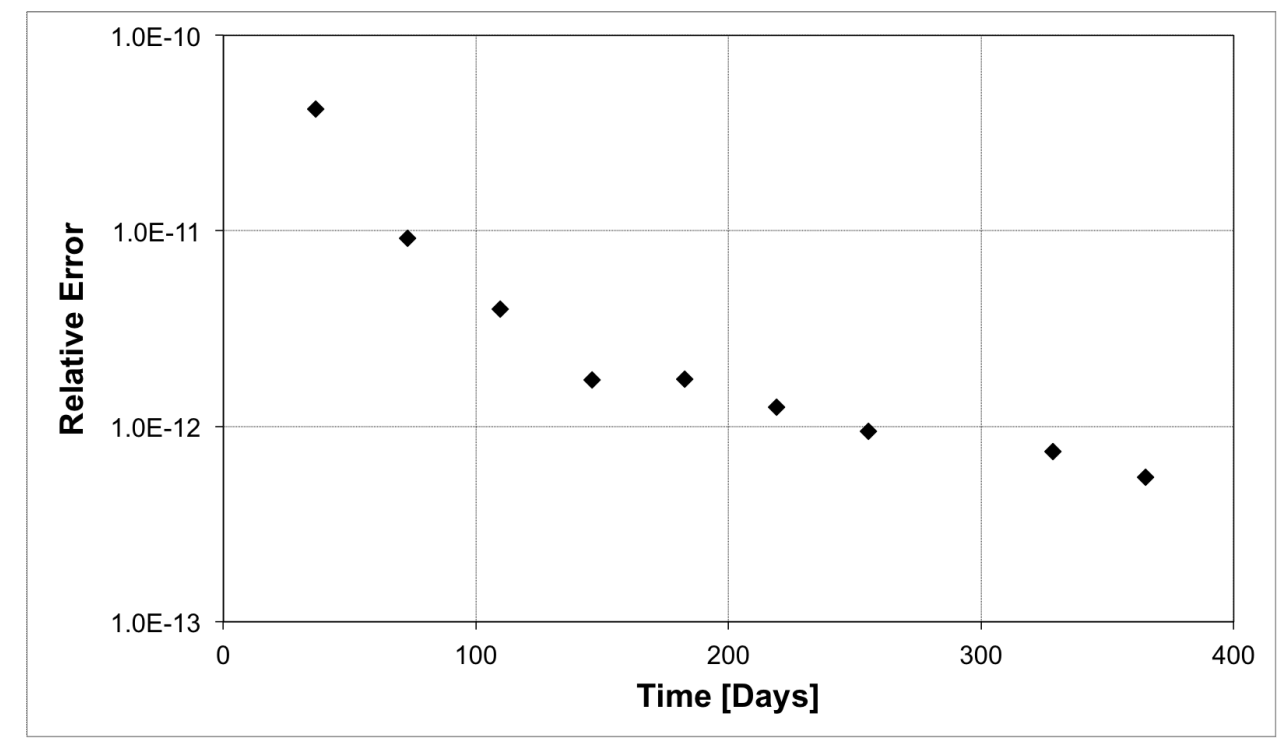

Figure 5: Relative Error between the Reference and MAMMOTH solutions for Pb-208 concentration for the Thorium series. 
Table 10: MAMMOTH solution number densities for isotopes in the thorium-232 series after 1 year.

\begin{tabular}{|c|c|c|c|c|}
\hline Time (days) & Th-232 & $\mathrm{Ra}-228$ & Ac- 228 & Th-228 \\
\hline 36.5 & $6.022999999970 \mathrm{E}+23$ & $2.953567638154 \mathrm{E}+12$ & $3.627339589142 \mathrm{E}+08$ & $1.726783939691 \mathrm{E}+10$ \\
\hline 73.0 & $6.022999999940 \mathrm{E}+23$ & $5.871744543953 \mathrm{E}+12$ & $7.248715742555 \mathrm{E}+08$ & $6.867693005509 \mathrm{E}+10$ \\
\hline 109.5 & $6.022999999911 \mathrm{E}+23$ & $8.754954782151 \mathrm{E}+12$ & $1.082669923668 \mathrm{E}+09$ & $1.525986405847 \mathrm{E}+11$ \\
\hline 146.0 & $6.022999999881 E+23$ & $1.160361733620 \mathrm{E}+13$ & $1.436181001830 \mathrm{E}+09$ & $2.674636649886 \mathrm{E}+11$ \\
\hline 182.5 & $6.022999999851 \mathrm{E}+23$ & $1.441814616915 \mathrm{E}+13$ & $1.785456180401 \mathrm{E}+09$ & $4.117635120054 \mathrm{E}+11$ \\
\hline 219.0 & $6.022999999821 E+23$ & $1.719895028378 \mathrm{E}+13$ & $2.130546215484 \mathrm{E}+09$ & $5.840482803809 \mathrm{E}+11$ \\
\hline 255.5 & $6.022999999792 \mathrm{E}+23$ & $1.994643378205 \mathrm{E}+13$ & $2.471501255008 \mathrm{E}+09$ & $7.829245140704 \mathrm{E}+11$ \\
\hline 292.0 & $6.022999999762 \mathrm{E}+23$ & $2.266099592383 E+13$ & $2.808370846007 \mathrm{E}+09$ & $1.007053134518 \mathrm{E}+12$ \\
\hline 328.5 & $6.022999999732 \mathrm{E}+23$ & $2.534303118491 \mathrm{E}+13$ & $3.141203941827 \mathrm{E}+09$ & $1.255147447261 \mathrm{E}+12$ \\
\hline 365.0 & $6.022999999702 \mathrm{E}+23$ & $2.799292931430 \mathrm{E}+13$ & $3.470048909235 \mathrm{E}+09$ & $1.525971220200 \mathrm{E}+12$ \\
\hline Time (day & $\mathrm{Ra}-224$ & $\mathrm{Rn}-220$ & Po-216 & $\mathrm{Pb}-212$ \\
\hline 36.5 & $6.770295695165 \mathrm{E}+07$ & $1.199525306803 \mathrm{E}+04$ & $3.128258654684 \mathrm{E}+01$ & $7.939993206433 \mathrm{E}+06$ \\
\hline 73.0 & $102328977781 \mathrm{E}+08$ & $5.496713712634 \mathrm{E}+$ & $1.433495695290 \mathrm{E}+02$ & $3.717037958896 \mathrm{E}+07$ \\
\hline 109.5 & 7.231989443710E+08 & $1.281377826921 \mathrm{E}+05$ & $3.341723329184 \mathrm{E}+02$ & $8.722319214698 \mathrm{E}+07$ \\
\hline 146.0 & $1.298389797172 \mathrm{E}+09$ & & & $1.570954782267 \mathrm{E}+08$ \\
\hline 182.5 & $2.027908498992 \mathrm{E}+09$ & $3.593113727562 \mathrm{E}+05$ & $9.370532207861 \mathrm{E}+02$ & $2.458226146684 \mathrm{E}+08$ \\
\hline 219.0 & $904164707129 E+09$ & $5.145701970347 E+05$ & $1.341954915050 \mathrm{E}+03$ & $3.524774300960 \mathrm{E}+08$ \\
\hline 255.5 & $3.919863473830 \mathrm{E}+09$ & 6.94536185 & 1.81129077431 & $4.761688345706 \mathrm{E}+08$ \\
\hline 292.0 & $5.067994426380 \mathrm{E}+09$ & 8.97967203 & $2.341821418979 \mathrm{E}+03$ & $6.160404979929 \mathrm{E}+08$ \\
\hline 328.5 & $6.341821336097 \mathrm{E}+09$ & 366969 & $9 \mathrm{E}+03$ & $7.712695760089 \mathrm{E}+08$ \\
\hline 365.0 & $7.734872062335 \mathrm{E}+09$ & $1.370496881064 \mathrm{E}+06$ & $3.574138286889 \mathrm{E}+03$ & $9.410654817206 \mathrm{E}+08$ \\
\hline Time (day & Bi-212 & Po-212 & Tl-208 & $\mathrm{Pb}-208$ \\
\hline 36.5 & $.501737681478 \mathrm{E}+05$ & 5073325532E-05 & $150693722 \mathrm{E}+04$ & $1.310116157101 \mathrm{E}+08$ \\
\hline & $3.519211116437 \mathrm{E}+06$ & $1.855401855480 \mathrm{E}$ & 6713399333E+04 & $1.313896772166 \mathrm{E}+09$ \\
\hline 109.5 & $8.263344020324 \mathrm{E}+06$ & $4.356608148960 \mathrm{E}-04$ & $1.497343094422 \mathrm{E}+05$ & $4.761869212371 \mathrm{E}+09$ \\
\hline 6.0 & $1.488744026560 \mathrm{E}+07$ & 7.848970515902E-04 & $7691038057 \mathrm{E}+05$ & $1.163451205088 \mathrm{E}+10$ \\
\hline 182.5 & $2.330000268060 \mathrm{E}+07$ & $1.228424972983 \mathrm{E}-03$ & $4.222134591462 \mathrm{E}+05$ & $2.303525827426 \mathrm{E}+10$ \\
\hline 219.0 & $3.341308648480 \mathrm{E}+07$ & $1.761607945931 \mathrm{E}-03$ & $6.054736918562 \mathrm{E}+05$ & $4.001356399743 \mathrm{E}+10$ \\
\hline 255.5 & $4.514216930888 \mathrm{E}+07$ & 2.379989773984E-03 & $8.180181372396 \mathrm{E}+05$ & $6.356700654221 E+10$ \\
\hline 292.0 & $5.840602583818 \mathrm{E}+07$ & 3.079288088323E-03 & $1.058374877876 \mathrm{E}+06$ & $9.464330569818 \mathrm{E}+10$ \\
\hline & $7.312660696190 \mathrm{E}+07$ & 3.855387976254E-03 & $1.325129553631 \mathrm{E}+06$ & $1.341422709221 \mathrm{E}+11$ \\
\hline 365.0 & $8.922892326708 \mathrm{E}+07$ & 4.704335838762E-03 & $1.616923250406 \mathrm{E}+06$ & $1.829176771397 \mathrm{E}+11$ \\
\hline
\end{tabular}


Table 11: Relative Error between Reference and MAMMOTH solutions for $\mathrm{Pb}-208$ for Thorium Series

\begin{tabular}{c|c} 
Time [Days] & Relative Error \\
\hline 36.5 & $4.198103859300 \mathrm{E}-11$ \\
73 & $9.133201464106 \mathrm{E}-12$ \\
109.5 & $3.990041002454 \mathrm{E}-12$ \\
146 & $1.719062877426 \mathrm{E}-12$ \\
182.5 & $1.736508227996 \mathrm{E}-12$ \\
219 & $1.249652536849 \mathrm{E}-12$ \\
255.5 & $9.438474746149 \mathrm{E}-13$ \\
292 & $0.000000000000 \mathrm{E}+00$ \\
328.5 & $7.454089157664 \mathrm{E}-13$ \\
365 & $5.465605484433 \mathrm{E}-13$ \\
\hline
\end{tabular}




\subsubsection{MAMMOTH-to-ORIGEN Thorium Series Comparison}

The percent difference for the thorium series runs in MAMMOTH and ORIGEN are shown in Table 13. The relative differences between the MAMMOTH and ORIGEN results are consistently below $0.03 \%$. ORIGEN and MAMMOTH both operate with double precision. The differences between the two results are expected to be differences in the conversion from years to seconds in either code.

Table 12: ORIGEN and MAMMOTH solutions for thorium series at 1 year

\begin{tabular}{c|c|c} 
Isotope & ORIGEN Number Density & MAMMOTH Number Density \\
\hline Th-232 & $6.023000 \mathrm{E}+06$ & $6.023000 \mathrm{E}+06$ \\
Ra-228 & $2.799553 \mathrm{E}-04$ & $2.799293 \mathrm{E}-04$ \\
Ac-228 & $3.412654 \mathrm{E}-08$ & $3.412246 \mathrm{E}-08$ \\
Th-228 & $1.526333 \mathrm{E}-05$ & $1.526056 \mathrm{E}-05$ \\
Ra-224 & $7.788272 \mathrm{E}-08$ & $7.786725 \mathrm{E}-08$ \\
Rn-220 & $1.369370 \mathrm{E}-11$ & $1.369092 \mathrm{E}-11$ \\
Po-216 & $3.571197 \mathrm{E}-14$ & $3.570474 \mathrm{E}-14$ \\
Pb-212 & $9.403015 \mathrm{E}-09$ & $9.401027 \mathrm{E}-09$ \\
$\mathrm{Bi}-212$ & $8.915940 \mathrm{E}-10$ & $8.914056 \mathrm{E}-10$ \\
Po-212 & $1.572066 \mathrm{E}-16$ & $1.571747 \mathrm{E}-16$ \\
Tl-208 & $1.615667 \mathrm{E}-11$ & $1.615269 \mathrm{E}-11$ \\
Pb-208 & $1.828924 \mathrm{E}-06$ & $1.828397 \mathrm{E}-06$ \\
\hline
\end{tabular}


Table 13: Relative Differences of Number Densities between ORIGEN and MAMMOTH Thorium Series

\begin{tabular}{c|c} 
Isotope & Absolute Relative Difference \\
\hline Th-232 & $0.000000 \mathrm{E}+00$ \\
Ra-228 & $9.287197 \mathrm{E}-05$ \\
Ac-228 & $1.195550 \mathrm{E}-04$ \\
Th-228 & $1.814807 \mathrm{E}-04$ \\
$\mathrm{Ra}-224$ & $1.986320 \mathrm{E}-04$ \\
$\mathrm{Rn}-220$ & $2.030131 \mathrm{E}-04$ \\
$\mathrm{Po}-216$ & $2.024531 \mathrm{E}-04$ \\
$\mathrm{~Pb}-212$ & $2.114215 \mathrm{E}-04$ \\
$\mathrm{Bi}-212$ & $2.113069 \mathrm{E}-04$ \\
$\mathrm{Po}-212$ & $2.029177 \mathrm{E}-04$ \\
$\mathrm{Tl}-208$ & $2.463379 \mathrm{E}-04$ \\
$\mathrm{~Pb}-208$ & $2.881476 \mathrm{E}-04$ \\
\hline
\end{tabular}

\subsection{Constant Flux in a Homogeneous System}

Both the analytic and the MAMMOTH solutions for the U-235, I-135 and Xe-135 transmutation chain test are shown in Figure 6. The I-135 and Xe-135 concentrations reach the saturation point during the constant flux depletion. At $100 \mathrm{hrs}$, the flux level is decreased to 0 and the I-135 continues to decay into Xe-135, thus creating a typical Xe transient event. 


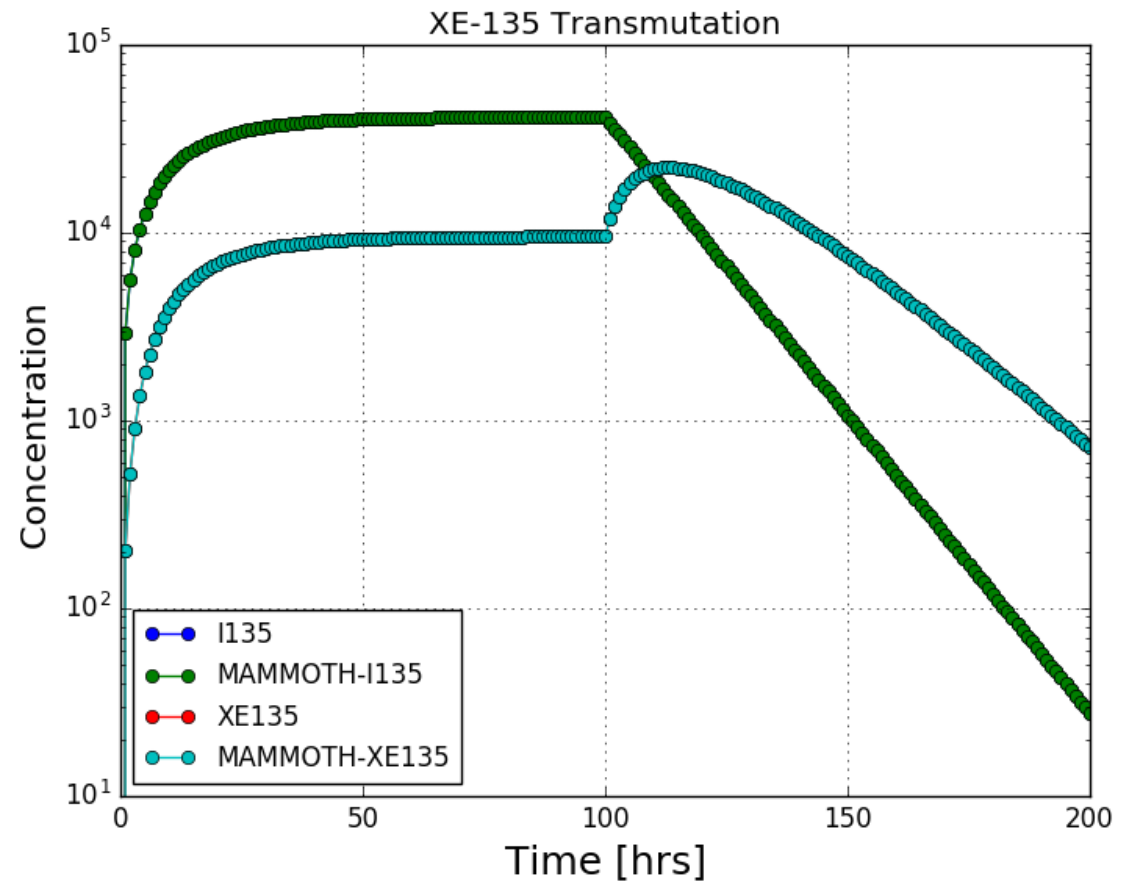

Figure 6: Reference and MAMMOTH solution for the Xe-135 chain. 


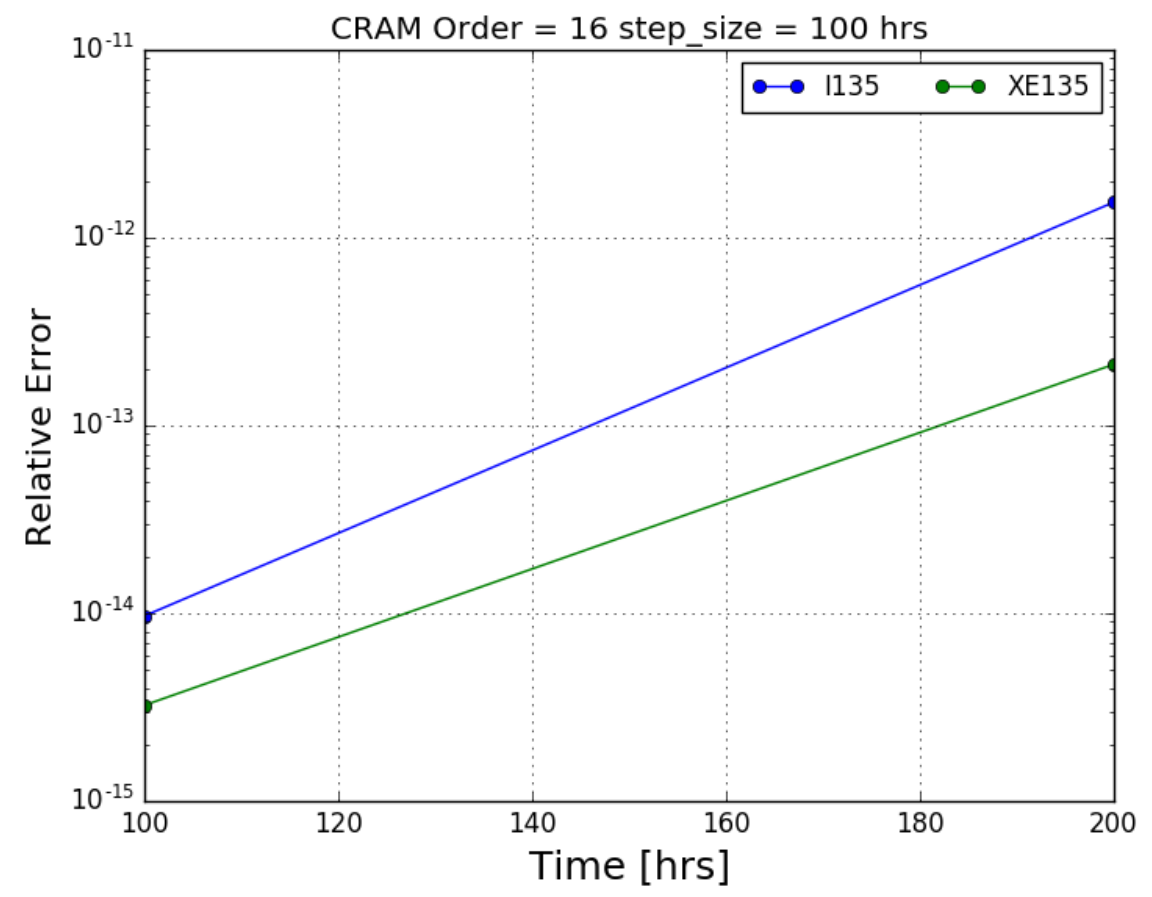

Figure 7: Relative error for the Xe-135 chain with large time steps. (Benchmark accuracy $\sim 1 \times 10^{-12}$ relative error)

\subsection{MAMMOTH-to-DRAGON5 Fuel Pin Comparison}

The results of the relative difference in final concentrations for select isotopes are shown in Figures 8 to 15 and the results of eigenvalue calculations are shown in Figures 12 and 13. These results indicate significant agreement between DRAGON-5 and MAMMOTH which is within the precision of the data used by DRAGON-5. 


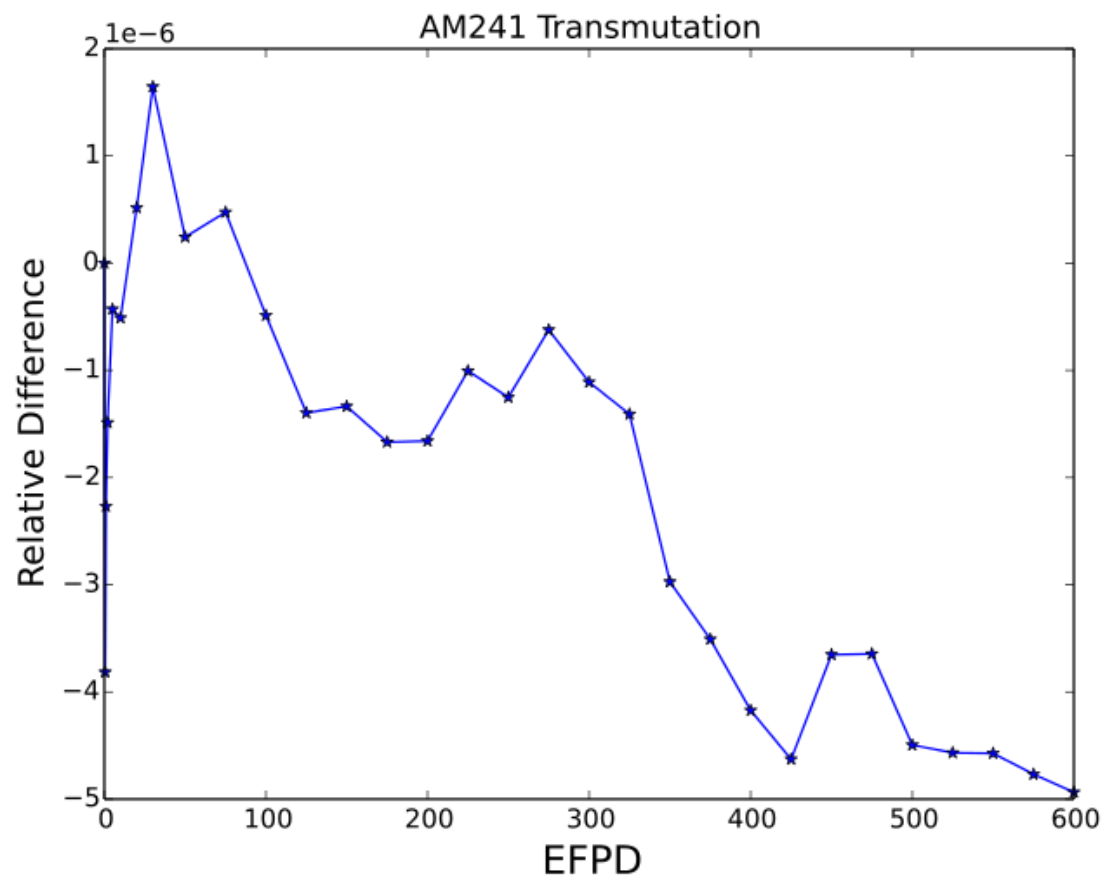

Figure 8: Relative difference between DRAGON-5 and MAMMOTH number density solutions for americium-241. 


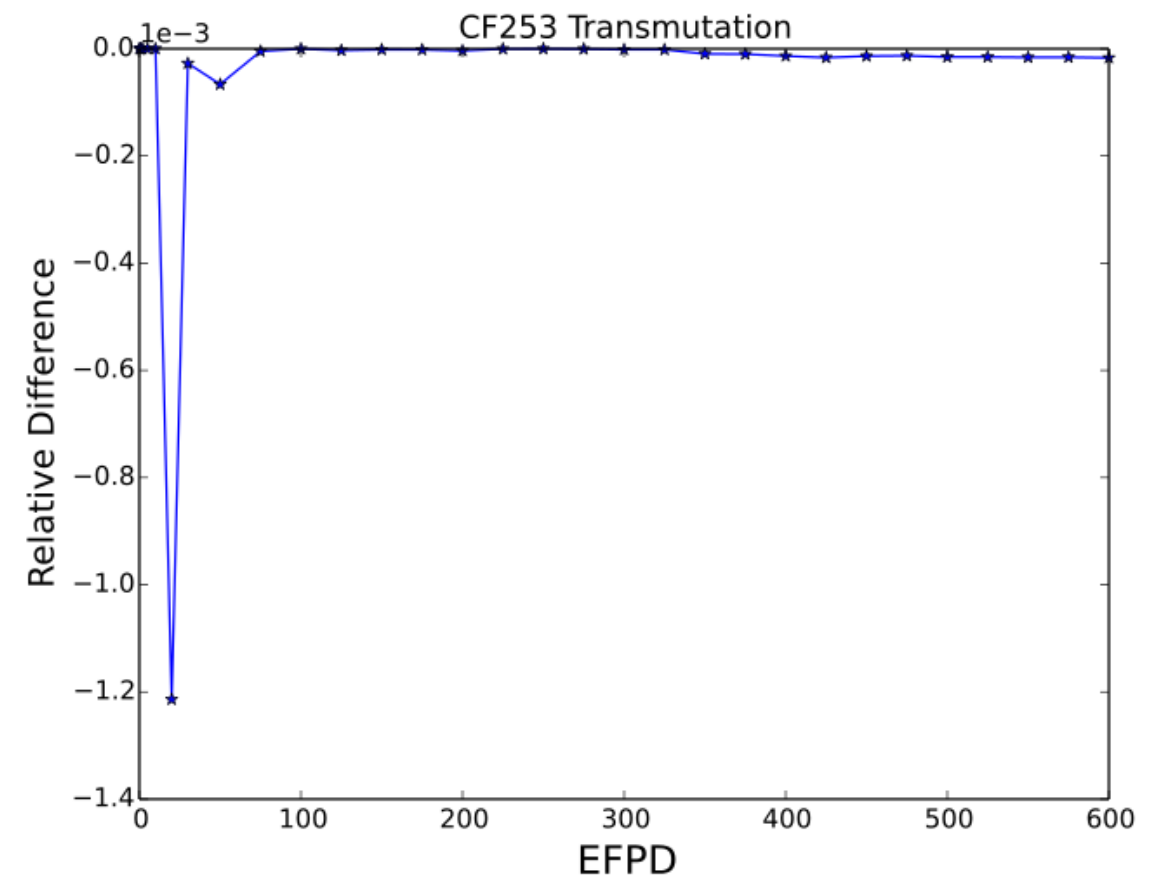

Figure 9: Relative difference between DRAGON-5 and MAMMOTH number density solutions for californium-253. 


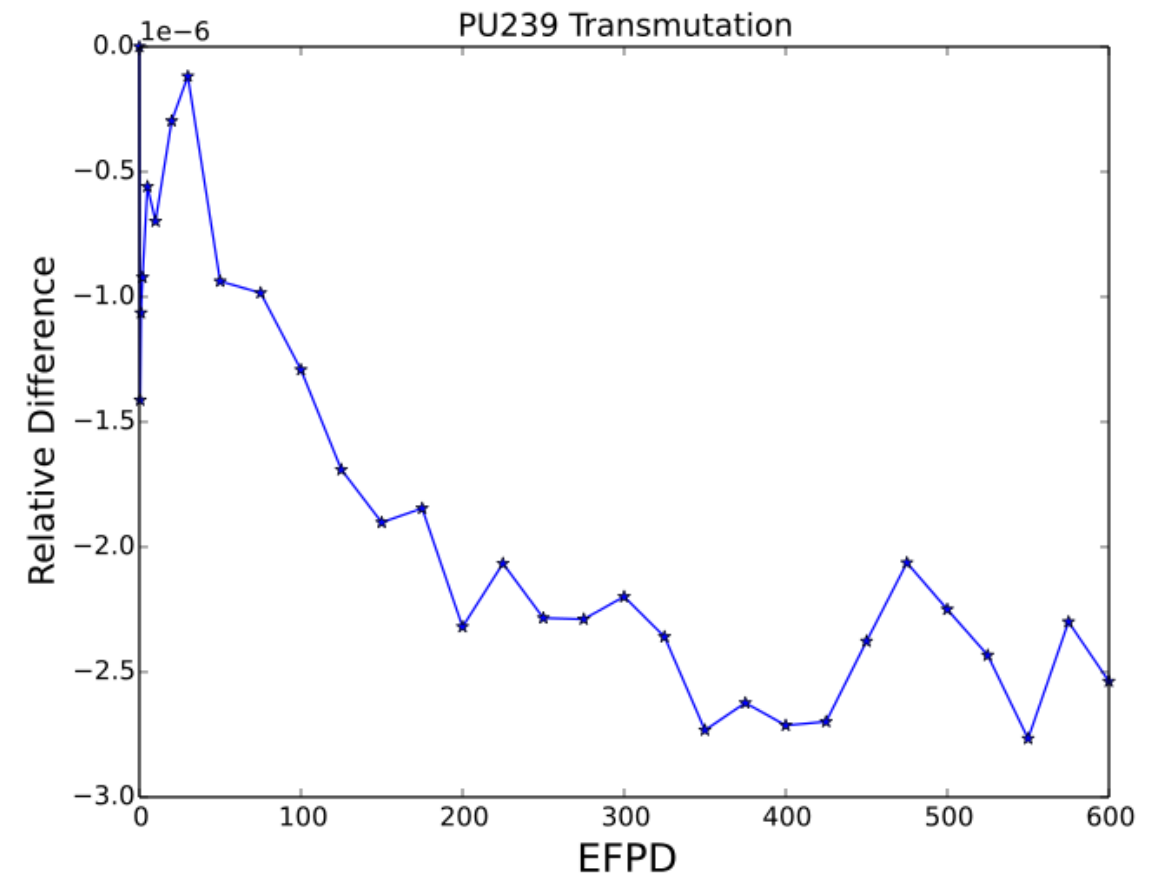

Figure 10: Relative difference between DRAGON-5 and MAMMOTH number density solutions for plutonium-239. 


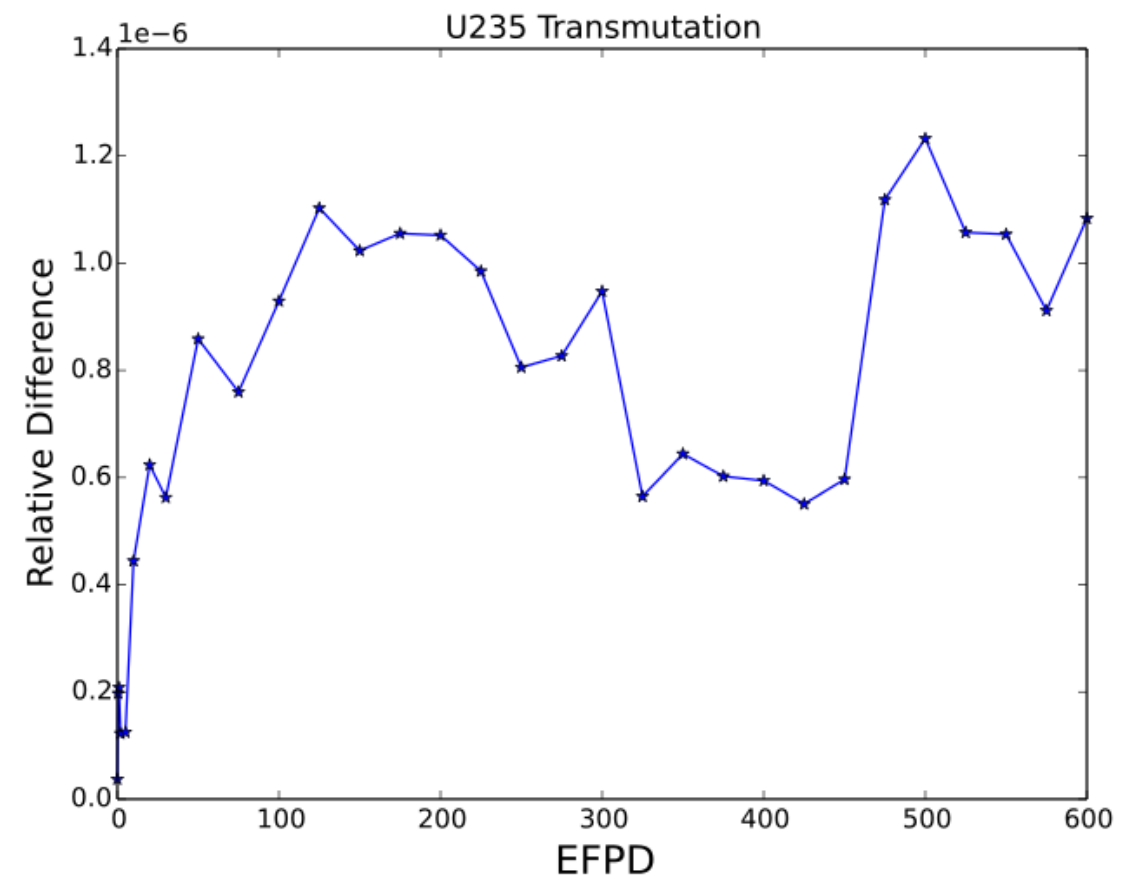

Figure 11: Relative difference between DRAGON-5 and MAMMOTH number density solutions for uranium-235. 


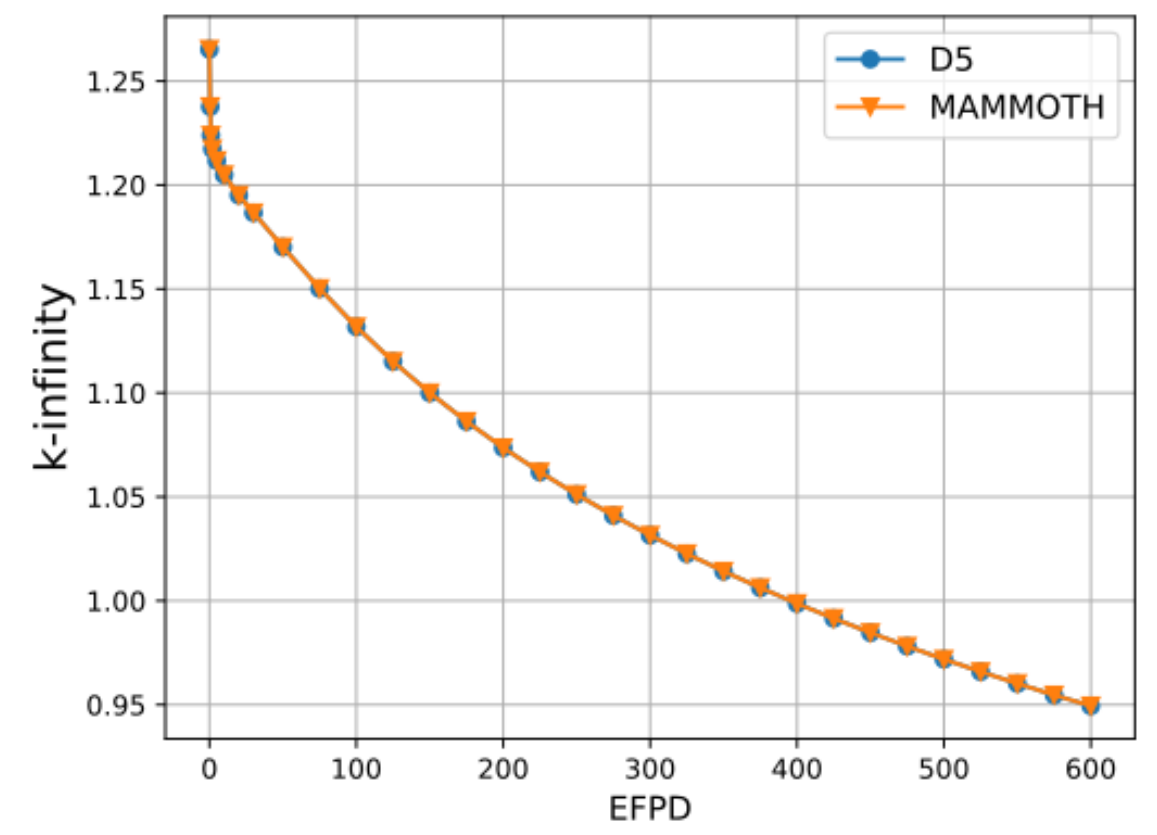

Figure 12: Eigenvalue results from DRAGON-5 and MAMMOTH. 


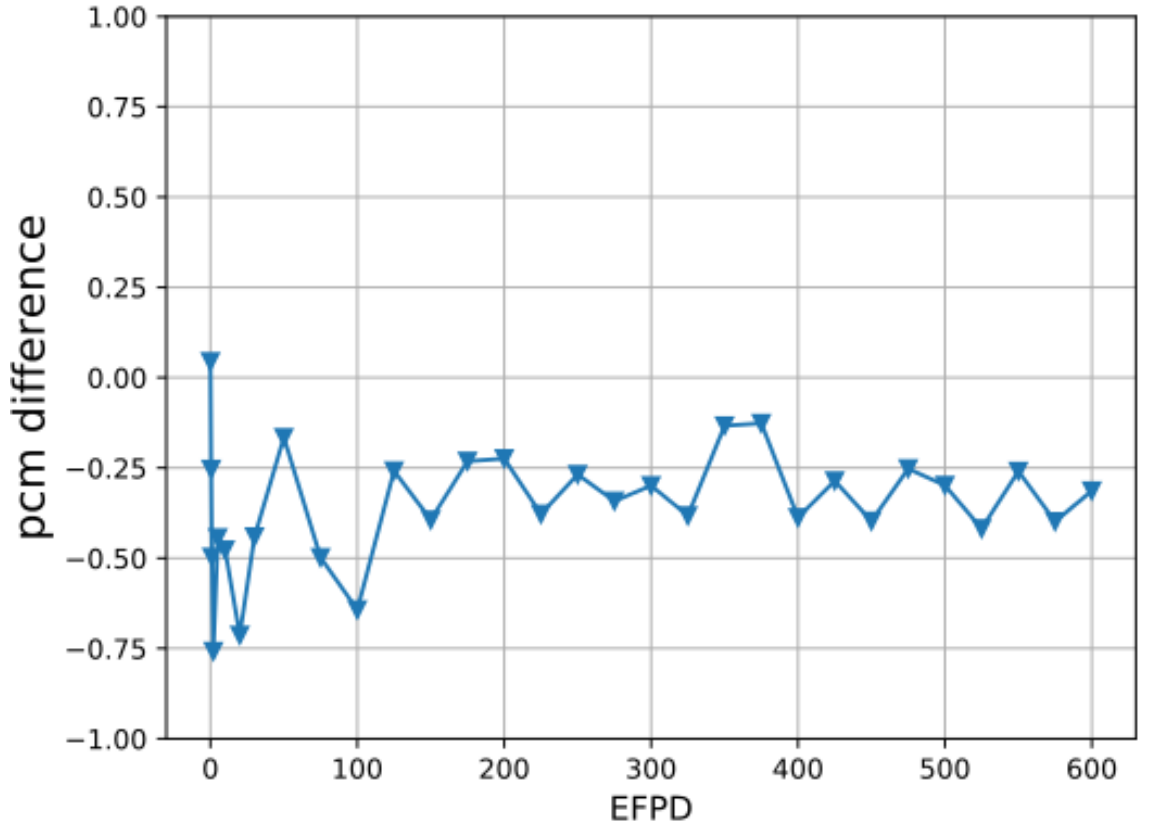

Figure 13: Per cent mille difference between the DRAGON-5 and MAMMOTH results. 


\subsection{MAMMOTH-to-ORIGEN Fuel Pin Comparison}

The relative error in concentrations for select isotopes between ORIGEN and MAMMOTH are shown in Figures 14 to 17 , the relative error of the scalar neutron flux tracked by both ORIGEN and MAMMOTH is shown in Figure 18. Maximum observed differences between MAMMOTH and ORIGEN were consistently below $2 \%$, which occur at roughly the one day mark for plutonium-239 and xenon-135. Upon further investigation, a comparison had been made using the ORIGEN matrix solver and CRAM [14]. In this comparison, PWR fuel, both fresh and old, was depleted for a time periods of both 1 and 100 days, and numerous isotopes exhibited relative error greater than $1 \%$ for both the 1 and 100 day cases, particularly for isotopes with very low concentrations, of which both xenon-135 and plutonium-239 are when they exhibit their maximum error in the MAMMOTH-toORIGEN comparison. IN the same work, the CRAM comparison consistently exhibited error less than $0.1 \%$ for the vast majority of isotopes for all time periods tested, resulting in confidence in the results provided by MAMMOTH from CRAM as the ORIGEN method is known to be less accurate than CRAM. After 100 days, the relative error between ORIGEN and MAMMOTH consistently and steadily increased over time for all isotopes, which is to be expected as compounding numerical errors result in the result becoming less and less physical.

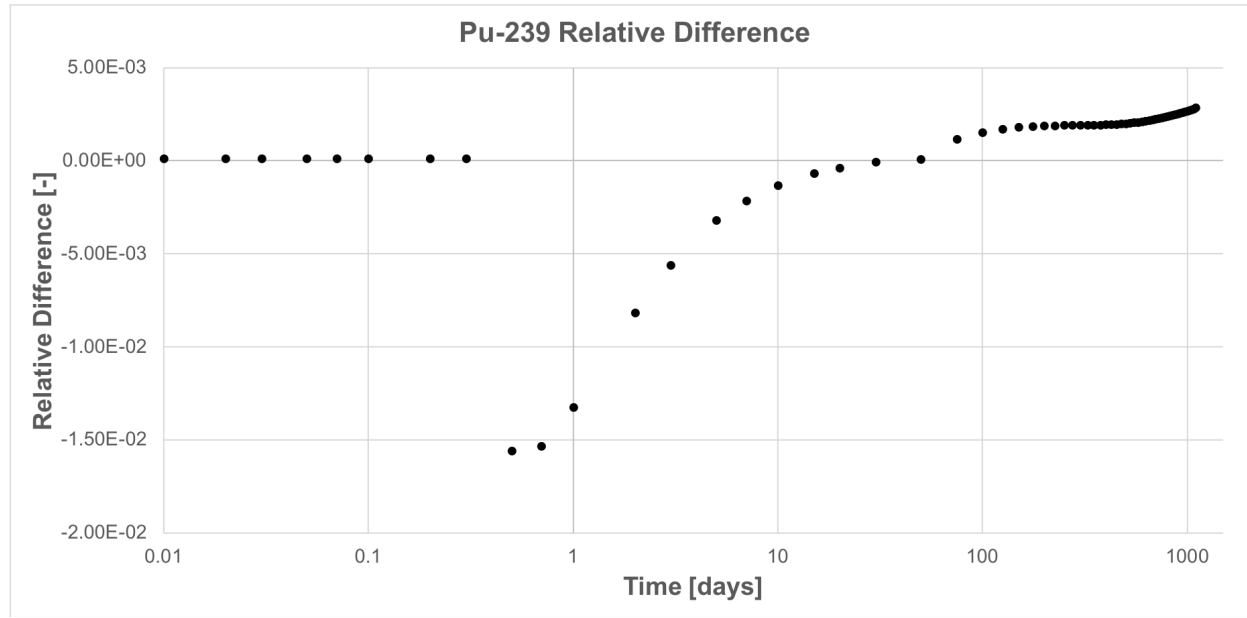

Figure 14: Relative error for the for the plutonium-239 concentration between ORIGEN and MAMMOTH. 


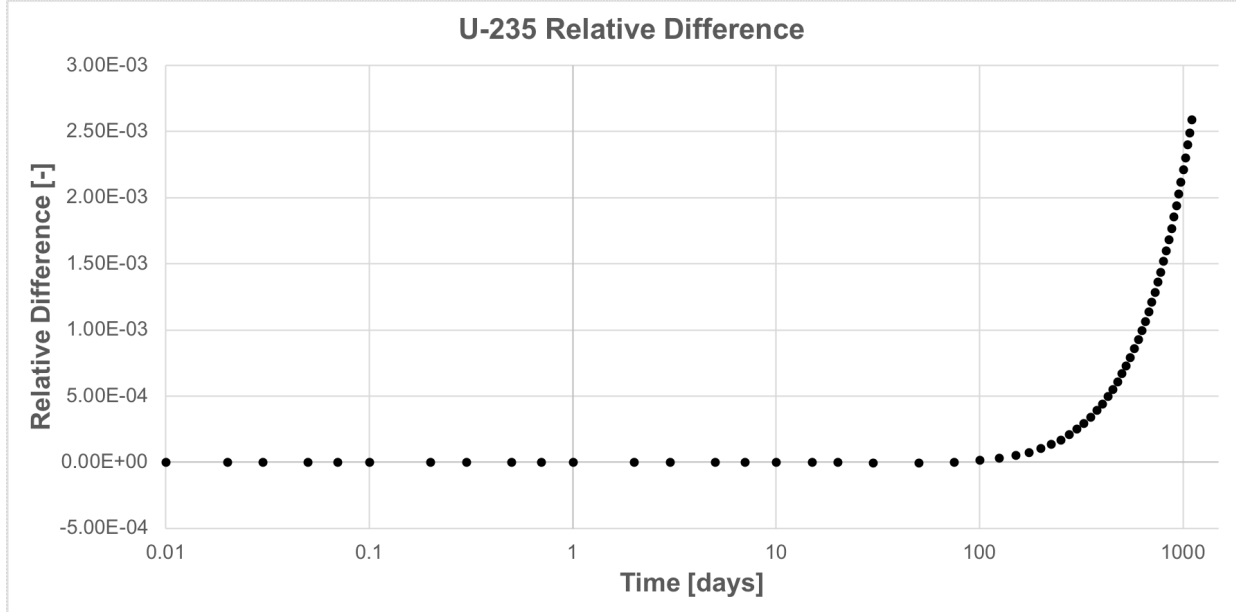

Figure 15: Relative error for the for the uranium-235 concentration between ORIGEN and MAMMOTH.

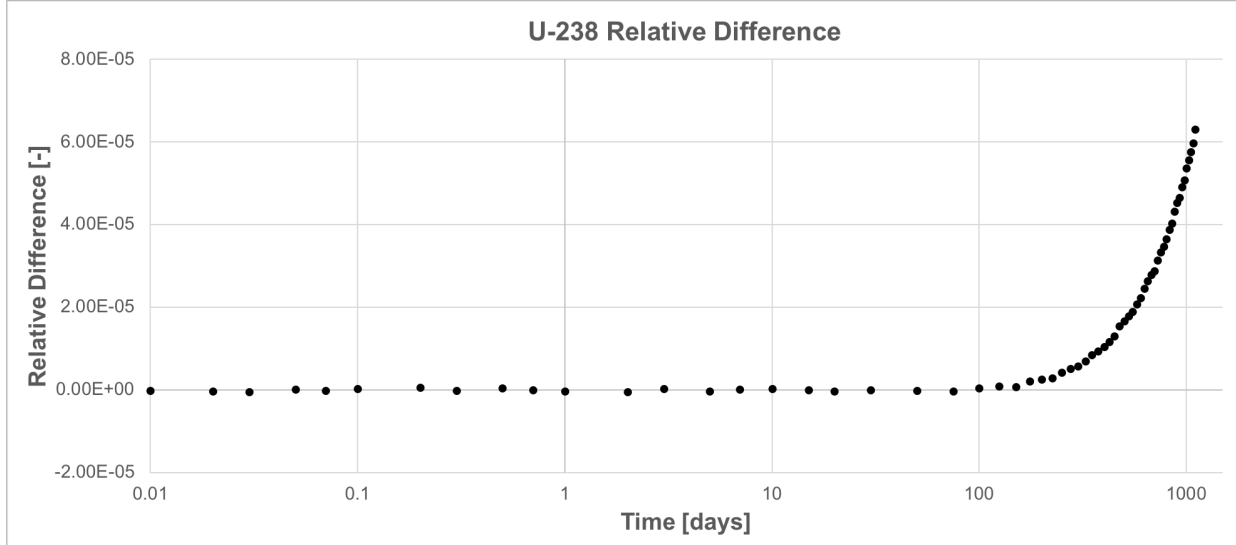

Figure 16: Relative error for the for the uranium-238 concentration between ORIGEN and MAMMOTH. 


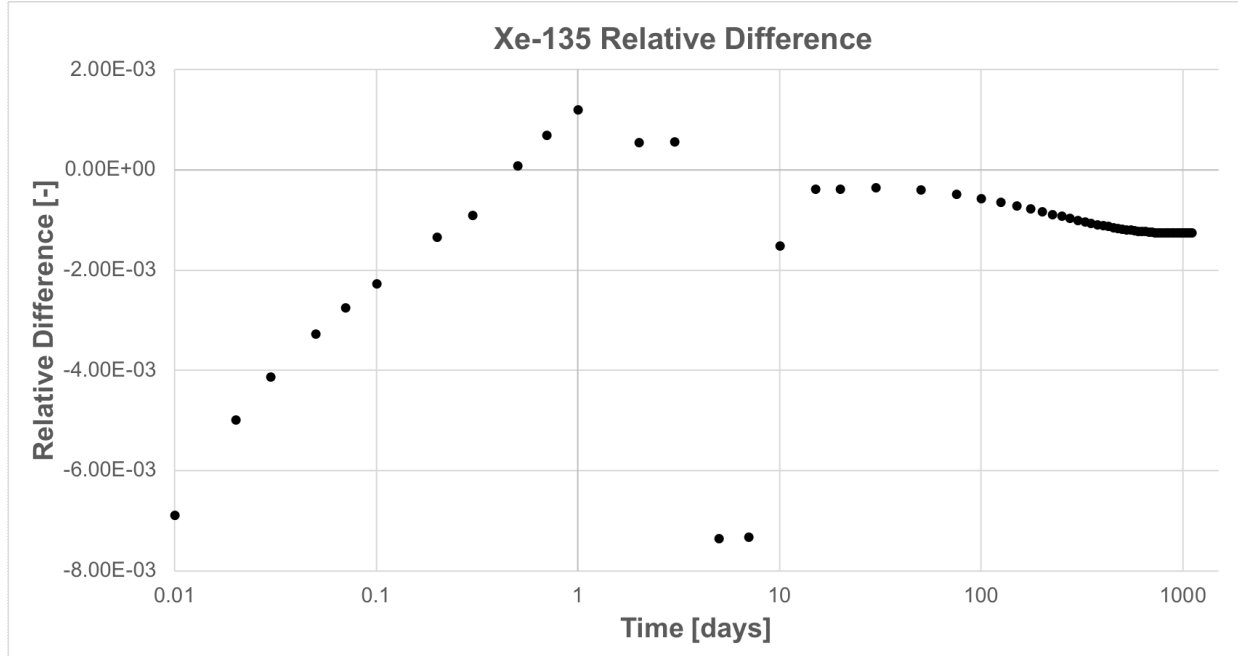

Figure 17: Relative error for the for the xenon-135 concentration between ORIGEN and MAMMOTH. 


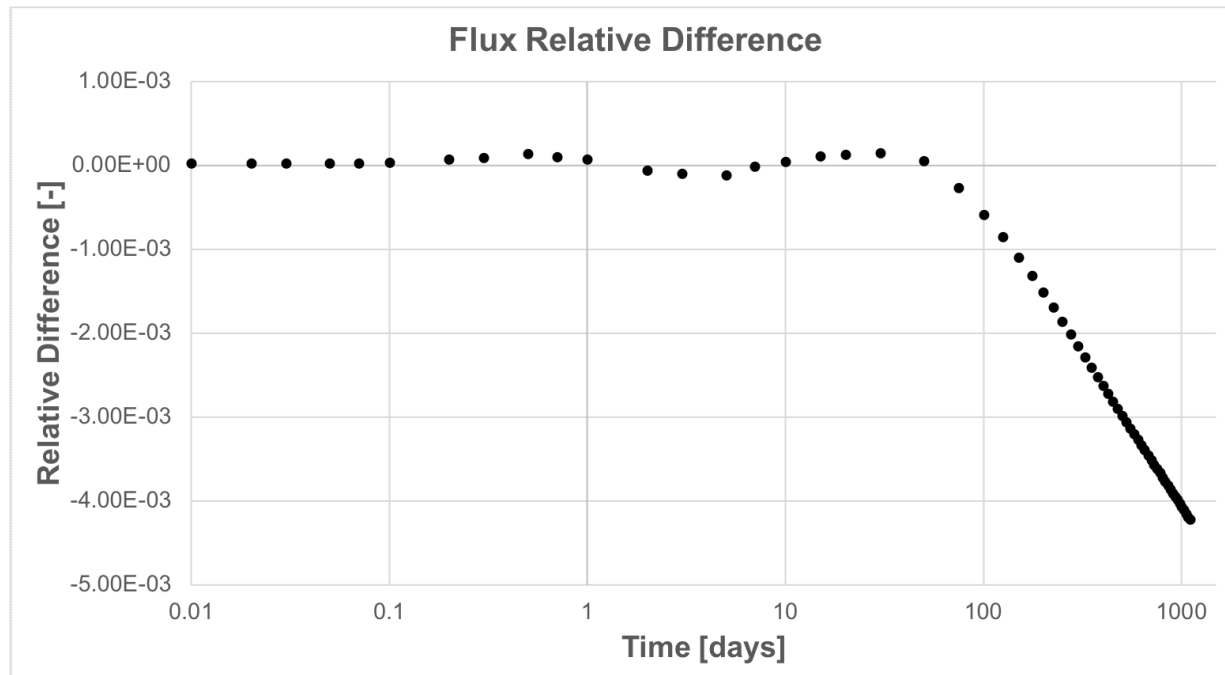

Figure 18: Relative error for the for the scalar neutron flux between ORIGEN and MAMMOTH. 


\section{Conclusions}

The implementation of data loaders to handle the ISOXML format as well as ORIGEN ENDF formatted decay data files in MAMMOTH was successful. The data loaders allow MAMMOTH to execute decay and transmutation problems using the ORIGEN transmutation and decay library encompassing more than 1,600 isotopes. The ISOXML format, which is documented here, allows users a greater degree of freedom to manipulate the decay data to suit specific user needs than the ORIGEN-formatted data. The implementation of the new depletion architecture, namely the creation of the Depletion User Object and the Working Decay Transmutation Library, has been successful and retesting of the thorium and xenon benchmarks and the DRAGON5 fuel pin problem verify this. The implementation of constant power depletion functionality has been successfully implemented and verified through comparison to ORIGEN.

Future work will include:

- the implementation of linear interpolation of fission product yield fractions based on incident neutron energy,

- the addition of the fission product yield fractions for spontaneous fission events of select nuclei into the ISOXML data format,

- the depletion of an infinite domain at a fixed flux level with one and multiple energy groups,

- the depletion of an infinite domain at a fixed power level with one and multiple energy groups,

- the depletion of a heterogeneous fuel cell at a constant flux with one and multiple energy groups,

- the depletion of a heterogeneous fuel cell at a fixed power level with one and multiple energy groups,

- and the performance of multiple validation studies against measurements. 


\section{ACKNOWLEDGMENTS}

This work was funded under the Nuclear Energy Advanced Modeling and Simulation (NEAMS) program managed by the Department of Energy Office of Nuclear Energy, under DOE Idaho Operations Office Contract DE-AC07-05ID14517. Accordingly, the U.S. Government retains a nonexclusive, royalty-free license to publish or reproduce the published form of this contribution, or allow others to do so, for U.S. Government purposes. 


\section{References}

[1] F. N. Gleicher et al. "The Coupling of the Neutron Transport Application Rattlesnake to the Fuels Performance Application BISON". In:

International Conference on Reactor Physics (PHYSOR 2014). Kyoto, Japan, May 2014.

[2] Derek Gaston et al. "MOOSE: A Parallel Computational Framework for Coupled Systems of Nonlinear Equations". In: Nuclear Engineering and Design 239.10 (2009), pp. 1768-1778.

[3] Derek R. Gaston et al. "Physics-based multiscale coupling for full core nuclear reactor simulation”. In: Annals of Nuclear Energy 84 (2015), pp. 45-54. DOI: $10.1016 / j$.anucene.2014.09.060.

[4] R.L. Williamson et al. "Multidimensional Multi-physics Simulation of Nuclear Fuel Behavior”. In: Jou. Nucl. Mat. 423.149-163 (2012).

[5] D. Andrs et al. RELAP-7 Level 2 Milestone Report. Tech. rep. INL/EXT-12-25924. Idaho National Laboratory, 2012.

[6] Yaqi Wang et al. "Hybrid PN - SN with Lagrange multiplier and upwinding for the multiscale transport capability in Rattlesnake”. In: Progress in Nuclear Energy (Apr. 2017). DOI: $10.1016 / j \cdot$ pnucene.2017.03.020.

[7] Yaqi Wang, Sebastian Schunert, and Vincent Laboure. Rattlesnake Theory Manual. INL. 2017.

[8] F. Gleicher et al. "The Application of MAMMOTH for a Detailed Strongly Coupled Fuel Pin Simulation with a Station Blackout". In:

Top Fuel 2016 - LWR Fuels with Enhanced Safety Performance. ANS, 2016.

[9] J. Ortensi et al. Initial Testing of the Microscopic Depletion Implementation. INL/EXT-16-39930. Idaho National Laboratory, Sept. 2016.

[10] H. Bateman. "The Solution of a System of Differential Equations Occurring in the Theory of Radioactive Transformations". In: Proceedings of the Cambridge Philosophical Society, Mathematical and physical sciences. Vol. 15. University Press, 1910, pp. 423-427.

[11] Tosaka. Decay Chain 4n, Thorium Series. http://www. commons.wikimedia.org, 2010. 
[12] K. Huang, Y. Li, and B. Ganapol. "A Backward Euler Doubling Feasibility Study Based on Thorium Series Cascade". In:

Transactions of the American Nuclear Society. Vol. 114. American Nuclear Society, 2016, pp. 399-402.

[13] G. Marleau, A. Hébert, and R. Roy. A User Guide for Dragon Version5. IGE-335. École Polytechnique de Montréal, Mar. 2016.

[14] C.J. Díez et al. "Comparison of nuclear data uncertainty propagation methodologies for PWR burn-up simulations”. In: Annals of Nuclear Energy 77 (2015), pp. 101-114. ISSN: 0306-4549. DOI:

https://doi.org/10.1016/j.anucene.2014.10.022. URL: http: //wWw.sciencedirect.com/science/article/pii/s0306454914005611. 